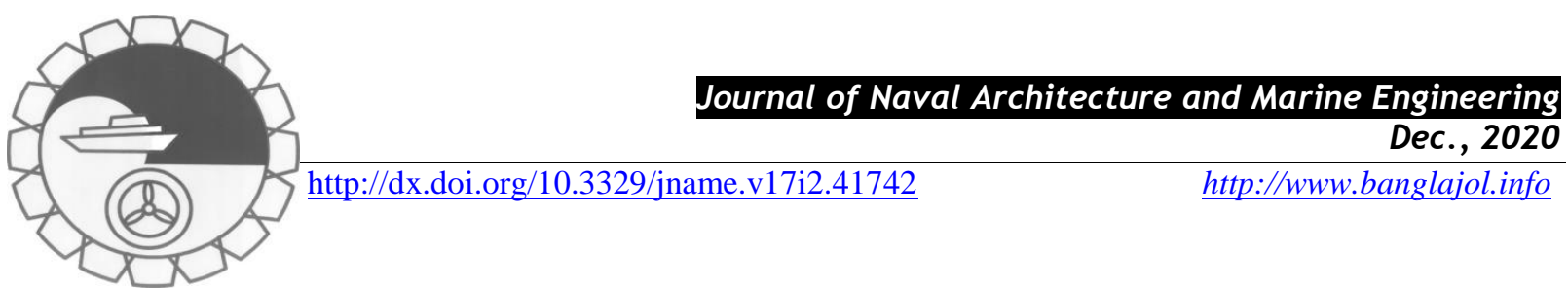

\title{
GRAVITATION MODULATION IMPACT ON MHD FREE CONVECTION FLOW OF MICROPOLAR FLUID
}

\author{
S. Sengupta ${ }^{*}$ and R. Deb ${ }^{2}$
}

${ }^{1 *}$ Department of Mathematics, Assam University, Silchar, Assam, India, E-mail: sanjib_aus2009@ @ediffmail.com; ${ }^{2}$ Department of Mathematics, Assam University, Silchar, Assam, India, ,E-mail: $\underline{\text { reshmideb27@ gmail.com }}$

\begin{abstract}
In this paper, a theoretical study is carried out on unsteady three dimensional, laminar, free convection flow of micropolar fluid with Hall effect, Joule heating and heat sink under gravitation modulation. A uniform transverse magnetic field is applied normal to the plate along the fluid region. The magnetic Reynolds number is considered to be small due to incomparability of applied and induced magnetic fields, as such the influence of induced magnetic field can be neglected. The multi parameter perturbation technique is used to solve the governed dimensionless equations. The fluid velocity, temperature and the concentration profiles are discussed with the aid of graphs and tables. The coefficient of skin friction and couple stresses is numerically computed in addition to Nusselt number and Sherwood number. The result reveals that the linear velocity increases due to escalation in gravitation modulation parameter values but for intensification in values of gravitation modulation parameter, a reverse effect is observed for rotational velocity. A comparative analysis shows that skin friction coefficient is less in micropolar fluid than the corresponding Newtonian fluid.
\end{abstract}

Keywords: Micropolar fluid, gravitation modulation, MHD, Hall current, Joule heating and heat sink.

\section{NOMENCLATURE}

$\bar{C}$
$\bar{C}$
$\bar{C}$
$\bar{C}$

$\bar{C}$

Species concentration

$\bar{C}_{m} \quad$ Species concentration at the plate

$C_{p} \quad$ Specific heat at constant pressure

$\bar{C}_{\infty} \quad$ Species concentration in the free stream

$D_{M} \quad$ Coefficient of mass diffusion

$\vec{g} \quad$ Acceleration due to gravity

Gm Thermal Grashof number

Gr Solutal Grashof number

$K \quad$ Permeability parameter

$k \quad$ Thermal conductivity

$K_{l} \quad$ Chemical reaction parameter

$N u_{R} \quad$ Nusselt number (real part)

$\mathrm{Pr} \quad$ Prandtl number

Ec Eckert number

$\mathrm{Q}_{\mathrm{s}} \quad$ Heat sink parameter

$s \quad$ Dimensionless material parameter

Sc Schmidt number

$S h_{R} \quad$ Sherwood number (real part)

$\mathrm{Sr} \quad$ Soret number

$M \quad$ Magnetic field parameter

$m_{0} \quad$ Hall parameter

$n \quad$ proportional parameter

$t \quad$ Time variable(non-dimensional)

$\bar{t} \quad$ Time variable (dimensional)
$\bar{T} \quad$ Fluid temperature

$\bar{T}_{m} \quad$ Mean Temperature

$\bar{T}_{\infty} \quad$ Temperature in the free stream

$u \quad$ First component of fluid velocity (non-dimensional)

$\bar{u} \quad$ First component of fluid velocity (dimensional)

$U_{R} \quad$ Real part of $U$

y $\quad$ y-Coordinate (non-dimensional)

$\bar{y} \quad \mathrm{y}$-Coordinate (dimensional)

$v \quad$ Second component of fluid velocity

$z \quad$ z- Coordinate (dimensional)

$w \quad$ Third component of fluid velocity

$\bar{v} \quad$ Second component of fluid velocity (dimensional)

\section{Greek Symbols}

$\theta \quad$ Non-dimensional temperature

$\rho \quad$ Fluid density

$\phi \quad$ Non-dimensional species concentration

$\omega \quad$ Frequency of oscillation

$\eta \quad$ Spin gradient viscosity

$v \quad$ Kinematic viscosity

$v_{r} \quad$ Rotational kinematic viscosity

$\alpha \quad$ Magnetic field inclination 


\begin{tabular}{llll} 
& parameter & $\theta_{R}$ & Real part of $\theta$ \\
$\xi$ & Vortex viscosity & $\phi_{R}$ & Real part of $\phi$ \\
$\sigma$ & Electrical conductivity & \multicolumn{2}{c}{ Subscripts } \\
$\beta_{T}$ & Thermal coefficient of volumetric & $m$ & Conditions on the wall \\
& expansion & $\infty$ & Free stream conditions \\
$\beta_{c}$ & Solutal coefficient of volumetric & & \\
& expansion & &
\end{tabular}

\section{Introduction}

The free convective magnetohydrodynamics micropolar fluid theory is constantly attracting a significant amount of researchers due to its profound impact in science and technical arenas. The concept of micropolar fluid theory developed by Eringen (1966) was a significant generalization of classical Navier-Stokes equation. Micropolar fluids are fluids with microstructure belonging to a class of fluids non-symmetrical stress tensor. The physical instances of micro polar fluids include polymers, lubricants, fluids with additives, paints, animal blood, colloidal and suspension solutions, etc. The theory of MHD flow models under the influence of various physical aspects is developing new approaches to deal with the flow related problems. The study of magnetohydrodynamics (MHD) has important applications found in metrology, planetary magnetospheres, and solar physics and in motion of the earth's core. A comprehensive review of the MHD free convection flow problem under Hall effect in flow domain was discussed by Chamkha (1997). Takhar et al. (2001) studied the boundary layer MHD flow induced by the impulsive stretching surface. Alam et al.(2010) analyzed the problem of unsteady twodimensional MHD convective flow of a micropolar fluid past a continuously moving porous plate under the influence of magnetic field. Gorla and Chamkha (2011) reported natural convection boundary layer flow over a non-isothermal vertical plate embedded in a porous medium. Alam and Hossain (2013) reported a new similarity approach for an unsteady two-dimensional flow of a micropolar fluid along a wedge. Rajput and Kumar (2019) analyzed the MHD flow past a vertical plate with variable wall temperature and mass diffusion under radiation and chemical reaction effects. Due to advancement of applied science, the expansion of free convective micropolar fluid flow including magnetohydrodynamical effects has great significance. A few illustrations of its various use in diverse spheres such as MHD generators, designing of cooling system of nuclear reactors, propulsion, nuclear generator, planetary magnetospheres and chemical science. Alam and Rahman (2005) analyzed MHD free convective heat and mass transfer flow with Soret and Dufor effect past a vertical porous flat plate embedded in a porous medium. Shamshuddin et al. (2018) studied MHD micropolar fluid with heat and mass transfer near to an inclined porous plate immersed in a porous medium. Sreenivasulu et al. (2018) investigated the MHD dissipative flow of a micropolar fluid under the effects of internal heat generation and viscous dissipation.

The Hall current effect plays a noteworthy role in case of strong magnetic flowing field since the electromagnetic strength creates notable effect in flowing field. Hall effect is a vital event which aids in determination of type of semiconductor. Hall effect devices procured extensive attention in flow field owing to their applications in geophysics, astrophysics and engineering section such as Hall effect sensor, turbine construction and centrifugal machines. A lots of analyst like T Srinivasacharya and Shiferaw (2009), Narayana et al. (2013), Reddy et al. (2014), Ayano and Demeke (2015), Singh (2017) etc. considered the case of Hall current effect on MHD micropolar fluid in the presence of various physical parameters and phenomena to examine the flow characteristics. In view of resolving various type of flow problems, the influence of Joule heating is relevant in designing a variety of electrical devices. Several researcher such as Eldabe and Ouaf (2006), Chamkha et al. (2011), Narayana et al. (2013) and Shamshuddin et al. (2018) analyzed the problem of Joule heating effect on MHD micropolar fluid by considering different physical factors under various sustainable conditions. The effect of heat source or heat sink in the flow process plays a vital role by designing several advanced energy convection materials. A significant use of heat source or heat sink effect was observed in welding engineering field. Several researchers like Khedr et al. (2009), Umavathi and Sultana (2012), Hayat et al. (2014), Sengupta and Sen (2013), Sengupta and Ahmed (2014,2016) and Chamkha et al. (2017) and so many .investigated heat sink effect with various flow parameters and conditions on flowing field of micropolar fluid In view of various fluid related difficulties in industrial and technological field due to rapid growth of mechanical science, the study of chemical reaction has become prominent. In many chemical science activities, chemical reactions take place between a foreign mass and the working fluid which moves due to stretching or otherwise of a surface. If the rate 
of chemical reaction is directly proportional to the concentration and it occurs as a single phase volume reaction the chemical reaction is then said to be of first order and homogenous. The chemical reaction possesses its applications in chemical industry, hydrometallurgical industry, petroleum industry, cooling of nuclear reactors and so many. The hydrogenation of ethene is an example of first order chemical reaction. In this case, the reaction between ethene and hydrogen in the presence of a metal crystal like nickel produce ethane. The rate is found to be directly proportional to first power of the concentration of the reactants. Kishan and Amrutha considered the effect of chemical reaction on nonlinear flow of MHD viscous electrically conducting fluid under the influence of thermal stratification and viscous dissipation. Rout et al. (2016) analyzed the impact of chemical reaction for free convective boundary layer flow of MHD micropolar fluid motion. Pattnaik et al. (2019) considered the chemical reaction effect with a second order slip flow on MHD micropolar fluid with boundary layer flow past a stretching sheet. The impact of gravity modulation in flow field is also now receiving a substantial amount of interest in current years because of its importance in a ample branch of technical applications. The time dependent gravity modulation is of realistic impact in diverse fields such as geophysics, astrophysics, geology, chemistry and metallurgy. It has been observed that a significant amount of analyst like Sharidan et al.(2005), Pranesh et al. (2014), Rajvanshi et al. (2014) Yekasi et al. (2016), Kumar and Pranesh (2018) has worked on the effect of gravity modulation under diverse physical circumstances in micropolar fluid. The aim of the present paper is to study the effect of gravitational modulation on laminar free convection flow of incompressible micropolar fluid in the presence of uniform magnetic field. The effect of Hall effect, Joule heatings, and heat sink are also considered in this study. A multi-parameter perturbation technique is used for solving purpose of the non-dimensional governed equations. The present study finds it potential applications in the field of microscale fluid mechanics and non-Newtonian fluid mechanics. The micropolar fluid models possess huge potential applications in diverse field. For example, in fluid mechanics, the fluid models are extensively used in turbo machinery design, designing of and piping and ducting used in water etc. In medical Science, the micropolar fluid model is used in cardiovascular problems, blood pressure measurement machine design, artificial breather machine and so on.

\section{Formulation of the Problem}

A three dimensional unsteady flow of free convective micropolar fluid past a vertical plate in the presence of Hall current, gravitational modulo and heat sink is considered for study. To generate the mathematical modeling of the flow situation, the $\bar{x}$ axis is taken along the length of the plate, $\bar{y}$-axis is considered perpendicular to the plate along the fluid region and $\bar{z}$ - axis is taken along width of the plate. Using the fundamental principle of mass conservation, conservation of linear momentum, conservation of angular momentum, energy conservation and species continuity, a fluid model is thus developed following the fluid model of Rajvanshi et al.(2014), Yekasi et al.(2016) as:

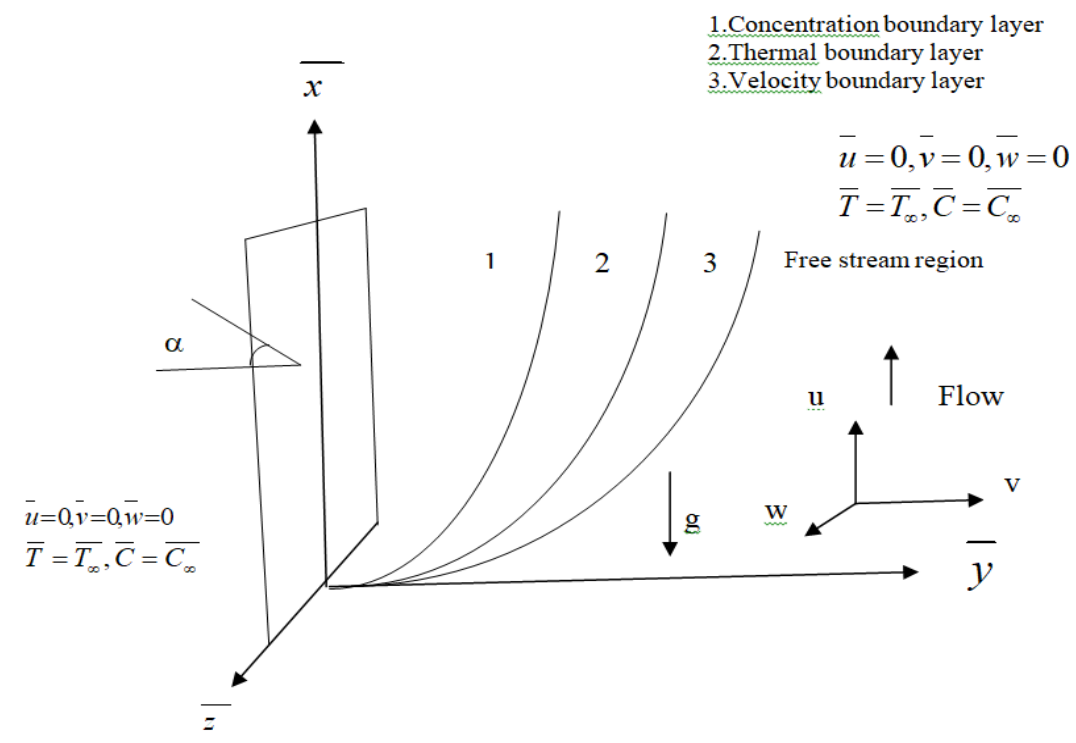

Fig. 1: Schematic representation of the flow domain and geometrical configuration 
The set of dynamical equations are:

$$
\begin{aligned}
& \frac{\partial \bar{w}}{\partial \bar{z}}=0 \\
& \frac{\partial \bar{u}}{\partial \bar{t}}+\bar{w} \frac{\partial \bar{u}}{\partial \bar{z}}=\left(v+v_{r}\right) \frac{\partial^{2} \bar{u}}{\partial \bar{z}}+g \beta_{T}\left(\bar{T}-\overline{T_{\infty}}\right)+g \beta_{C}\left(\bar{C}-\overline{C_{\infty}}\right)-2 v_{r} \frac{\partial \bar{N}_{2}}{\partial \bar{y}}+\frac{\sigma \mu_{e}^{2} H_{0}^{2} \sin ^{2} \alpha}{\rho\left(1+m_{0}{ }^{2}\right)}\left(m_{0} \bar{v}-\bar{u}\right) \\
& \frac{\partial \bar{v}}{\partial \bar{t}}+\bar{w} \frac{\partial \bar{v}}{\partial \bar{z}}=\left(v+v_{r}\right) \frac{\partial^{2} \bar{v}}{\partial \bar{z}^{2}}-\frac{\sigma \mu_{c}^{2} H_{0}^{2} \sin ^{2} \psi}{\rho\left(1+m^{2}\right)}\left(m_{0} \bar{u}+\bar{v}\right)+2 v_{r} \frac{\partial \overline{N_{1}}}{\partial \bar{z}} \\
& \rho I\left(\frac{\partial \overline{N_{i}}}{\partial \bar{t}}+\bar{w} \frac{\partial \overline{N_{i}}}{\partial \bar{z}}\right)=\gamma \frac{\partial^{2} \overline{N_{i}}}{\partial \bar{z}^{2}} \quad \forall \mathrm{i}=1,2 \\
& \rho C_{p}\left(\frac{\partial \bar{T}}{\partial \bar{t}}+\bar{w} \frac{\partial \bar{T}}{\partial \bar{z}}\right)=k \frac{\partial^{2} \bar{T}}{\partial \bar{z}^{-2}}+\frac{\sigma \mu_{c}^{2} H_{0}^{2} \sin ^{2} \psi}{\left(1+m_{0}{ }^{2}\right)}\left(\bar{u}^{2}+\bar{v}^{2}\right)-Q_{s}\left(\bar{T}-\bar{T}_{\infty}\right) \\
& \frac{\partial \bar{c}}{\partial \bar{t}}+\bar{w} \frac{\partial \bar{c}}{\partial \bar{z}}=D_{M} \frac{\partial^{2} \bar{c}}{\partial \bar{z}^{-2}}+\frac{D_{M} K_{T}}{C_{p} C_{s}} \frac{\partial^{2} \bar{T}}{\partial \bar{z}^{-2}}-\bar{K}_{l}\left(\bar{C}-\overline{C_{\infty}}\right)
\end{aligned}
$$

The micro polar fluid theory formulation possesses an additional factor of freedom gyration to characterize the rotational motion of microstructure. As a consequence, the balance equation of angular momentum (2.4) is provided for the purpose of solving gyration. In this way, the conservation of angular momentum equation provides a means for take into consideration the effect of molecular spin. The momentum equation is based on the law of conservation of momentum. It states that the net force $\left(F_{0}\right)$ acting on a fluid make a change in momentum of the flow per unit time in that direction. Mathematically, $F_{0}=\frac{d}{d t}\left(m_{0} v_{0}\right)$, where $m_{0}$ is the fluid mass, $v_{0}$ is the fluid velocity. The angular momentum equation states that the resulting torque on a rotating fluid is equal to the rate of change of momentum. Mathematically, $\tau_{0}=\frac{d}{d t}\left(I_{0} \omega_{0}\right)$, where $I_{0}$ is the rotational inertia, $\omega_{0}$ is angular velocity. The angular momentum equation is thus the rotational equivalent of the angular momentum equation.

Subject to the boundary conditions are:

$$
\begin{aligned}
& \bar{u}(\bar{z}, \bar{t})=0=\bar{v}(\bar{z}, \bar{t}), \bar{w}(\bar{z}, \bar{t})=0 \quad \bar{N}(\bar{z}, \bar{t})=0 \quad \bar{T}=\overline{T_{\infty}}, \bar{C}=\overline{C_{\infty}} \quad \text { at } \mathrm{t}>0 \\
& \bar{u}(0, \bar{t})=u_{p} \bar{v}(0, \bar{t})=0, \bar{w}(0, \bar{t})=-w_{0}\left(1+\varepsilon \delta \exp (\bar{i} \bar{\omega} \bar{t}), \bar{N}_{1}(0, \bar{t})=-n\left(\frac{\partial \bar{u}}{\partial \bar{t}}\right)_{\bar{z}=0} \bar{T}=\overline{T_{\infty}},\right. \\
& \bar{C}=\overline{C_{m}}+\varepsilon\left(\overline{C_{m}}-\overline{C_{\infty}}\right) e^{i \overline{\omega t}}, \bar{T}_{m}+\varepsilon\left(\bar{T}_{m}-\bar{T}_{\infty}\right) e^{i \bar{\omega} \bar{t}} \bar{N}_{2}(0, \bar{t})=-n\left(\frac{\partial \bar{v}}{\partial \bar{t}}\right)_{\bar{z}=0} \text { when } \bar{t}>0 \\
& , \quad \bar{t}>0
\end{aligned}
$$

where $g=g_{0}+g_{1} \exp (\bar{i} \overline{\omega t})$ with $g_{1}=\varepsilon \delta g_{0}$

$\mathrm{g}_{0}$ : some reference acceleration due to gravity.

We introduce the following non-dimensional quantities as:

$$
\begin{aligned}
& u=\frac{\bar{u}}{w_{0}}, \quad v=\frac{\bar{v}}{w_{0}}, w=\frac{\bar{w}}{w_{0}}, u_{p}=\frac{\overline{u_{p}}}{w_{0}}, z=\frac{\bar{z} w_{0}}{v}, t=\frac{\bar{t} w_{0}^{2}}{v}, w=\frac{\bar{w} v}{w_{0}^{2}} N_{i}=\frac{\overline{N_{i}} v}{w_{0}^{2}}, \xi=\frac{v_{r}}{v}, \theta=\frac{\bar{T}-\overline{T_{\infty}}}{\bar{T}_{w}-\overline{T_{\infty}}}, \phi=\frac{\bar{C}-\overline{C_{\infty}}}{\overline{C_{m}}-\overline{C_{\infty}}}, \\
& G m=\frac{g \beta_{c} v\left(\overline{C_{m}}-\overline{C_{\infty}}\right)}{w_{0}^{3}}, G r=\frac{g \beta_{T} v\left(\overline{T_{m}}-\overline{T_{\infty}}\right)}{w_{0}^{3}}, M^{2}=\frac{\sigma \mu_{e}^{2} H_{0}^{2} v}{w_{0}^{3}}, E c=\frac{w_{0}^{2}}{C_{p}\left(\overline{T_{m}}-\overline{T_{\infty}}\right)}, Q_{s}=\frac{\overline{Q_{s}} v}{\rho C_{p} w_{0}^{2}}, \operatorname{Pr}=\frac{\rho v C_{p}}{k}, \\
& S c=\frac{v}{D_{M}}, S r=\frac{D_{M} K_{T}\left(\overline{T_{m}}-\overline{T_{\infty}}\right)}{v C_{p} C_{s}\left(\overline{C_{m}}-\overline{C_{\infty}}\right)}, K_{l}=\frac{v \overline{K_{l}}}{w_{0}^{2}} .
\end{aligned}
$$


The equations to be solved are

$$
\begin{aligned}
& \frac{\partial u}{\partial t}+w \frac{\partial u}{\partial z}=(1+\xi) \frac{\partial^{2} u}{\partial z^{2}}+(G r \theta+G m \phi)(1+\varepsilon \delta \exp (i \omega t))-2 \xi \frac{\partial N_{2}}{\partial z}+\frac{M \sin ^{2} \alpha}{1+m_{0}^{2}}\left(m_{0} v-u\right) \\
& \frac{\partial u}{\partial t}+w \frac{\partial v}{\partial z}=(1+\xi) \frac{\partial^{2} v}{\partial z^{2}}+2 \xi \frac{\partial N_{1}}{\partial z}-\frac{M \sin ^{2} \alpha}{1+m_{0}^{2}}\left(m_{0} u+v\right) \\
& \frac{\partial N_{i}}{\partial t}+w \frac{\partial N_{i}}{\partial z}=\left(1+\frac{\xi}{2}\right) \frac{\partial^{2} N_{i}}{\partial z^{2}}, \quad \forall \mathrm{i}=1,2 \\
& \frac{\partial \theta}{\partial t}+w \frac{\partial \theta}{\partial z}=\frac{1}{\operatorname{Pr}} \frac{\partial^{2} \theta}{\partial z^{2}}+\frac{M^{2} \sin ^{2} \alpha E c\left(u^{2}+v^{2}\right)}{1+m_{0}^{2}}-Q_{s} \theta \\
& \frac{\partial \phi}{\partial t}+w \frac{\partial \phi}{\partial z}=\frac{1}{S c} \frac{\partial^{2} \phi}{\partial z^{2}}+\frac{1}{S r} \frac{\partial^{2} \theta}{\partial z^{2}}-K_{l} \phi
\end{aligned}
$$

Also $u \rightarrow 0, v \rightarrow 0, w \rightarrow 0, N_{\mathrm{i}} \rightarrow 0, \theta \rightarrow 0, \phi \rightarrow 0, \forall t>0, z \rightarrow \infty$

The reduced form of equations is:

$$
\begin{aligned}
& \frac{\partial U}{\partial t}+w \frac{\partial U}{\partial z}=(1+\xi) \frac{\partial^{2} U}{\partial z^{2}}+(G r \theta+G m \phi)(1+\varepsilon \delta \exp (i w t))+2 i \xi \frac{\partial N}{\partial z}-\frac{M \sin ^{2} \alpha}{1+m_{0}{ }^{2}} U\left(1+i m_{0}\right) \\
& \frac{\partial N}{\partial t}+w \frac{\partial N}{\partial z}=\left(1+\frac{\xi}{2}\right) \frac{\partial^{2} N}{\partial z^{2}}-2 \xi N \\
& \frac{\partial \theta}{\partial t}+w \frac{\partial \theta}{\partial z}=\frac{1}{\operatorname{Pr}} \frac{\partial^{2} \theta}{\partial z^{2}}+\frac{M^{2} \sin ^{2} \alpha E c}{1+m_{0}^{2}} \bar{U}-Q_{s} \theta \\
& \frac{\partial \phi}{\partial t}+w \frac{\partial \phi}{\partial z}=\frac{1}{S c} \frac{\partial^{2} \phi}{\partial z^{2}}+S r \frac{\partial^{2} \theta}{\partial z^{2}}-K_{l} \phi
\end{aligned}
$$

Subject to the non-dimensional boundary conditions as:

$u(0, t)=u_{p}, w=-(1+\varepsilon \delta \exp (\mathrm{i} \omega t)), N=-n\left(\frac{\partial U}{\partial z}\right)_{z=0}$

$\theta(0, t)=1+\varepsilon \exp (\mathrm{i} \omega t), \phi(0, t)=1+\varepsilon \exp (\mathrm{i} \omega t), t>0$

and $U(\infty, t)=0, w(\infty, t)=0, N(\infty, t)=0, \theta(\infty, t)=0, \phi(\infty, t)=0, t>0$

where $U(z, t)=u(z, t)+i v(z, t)$ and $N(z, t)=N_{1}(z, t)+i N_{2}(z, t)$

\section{Method of solution:}

We first take trial form of solution as:

$$
f(z, t)=f_{0}(z)+\varepsilon f_{1}(z) \exp (i \omega t)
$$

Where, $f$ stands for $U, N, \theta$ and $\phi$ respectively.

On using the above solution form in equations from (2.17)-(2.23), we obtain

$$
\begin{aligned}
& (1+\xi) \frac{d^{2} U_{0}}{d z^{2}}+\frac{d U_{0}}{d z}-\frac{M^{2} \sin ^{2} \alpha}{1+m_{0}^{2}}\left(1+i m_{0}\right) U=-2 \xi i \frac{d N_{0}}{d z}-G r \theta_{0}-G m \phi_{0} \\
& \left(1+\frac{\xi}{2}\right) \frac{d^{2} N_{0}}{d z^{2}}+\frac{d N_{0}}{d z}-2 \xi N_{0}=0 \\
& \frac{1}{\operatorname{Pr}} \frac{d^{2} \theta_{0}}{d z^{2}}+\frac{d \theta_{0}}{d z}-Q_{s} \theta_{0}=\frac{M^{2}}{1+m_{0}^{2}} E c \sin ^{2} \alpha U_{0} \overline{U_{0}}
\end{aligned}
$$


$\frac{1}{S c} \frac{d^{2} \phi_{0}}{d z^{2}}+\frac{d \phi_{0}}{d z}-K_{l} \phi_{0}=-S r \frac{d^{2} \theta_{0}}{d z^{2}}$

$(1+\xi) \frac{d^{2} U_{1}}{d z^{2}}+\delta \frac{d U_{1}}{d z}-\left(\frac{M^{2} \sin ^{2} \alpha}{1+m_{0}^{2}}\left(1+i m_{0}\right)+i \omega\right) U_{1}=-2 \xi i \frac{d N_{1}}{d z}-\delta\left(G r \theta_{0}+G m \phi_{0}\right)-\left(G r \theta_{1}+G m \phi_{1}\right)$

$\left(1+\frac{\xi}{2}\right) \frac{d^{2} N_{1}}{d z^{2}}+\delta \frac{d N_{1}}{d z}-(2 \xi+i \omega) N_{1}=0$

$\frac{1}{\operatorname{Pr}} \frac{d^{2} \theta_{1}}{d z^{2}}+\delta \frac{d \theta_{1}}{d z}-\left(Q_{s}+i \omega\right) \theta_{1}=-\frac{M^{2}}{1+m_{0}^{2}} E c \sin ^{2} \alpha\left(U_{0} \bar{U}_{1}+U_{1} \bar{U}_{0}\right)$

$\frac{1}{S c} \frac{d^{2} \phi_{1}}{d z^{2}}+\delta \frac{d \phi_{1}}{d z}-\left(K_{l}+i \omega\right) \phi_{1}=-S r \frac{d^{2} \theta_{1}}{d z^{2}}$

Subject to the set of boundary conditions as;

$U_{0}=u_{p}, N_{0}=-n\left(\frac{\partial U_{0}}{\partial z}\right)_{z=0} \theta_{0}=1, \phi_{0}=1$ at $z=0$

$U_{0} \rightarrow 0, N_{0} \rightarrow 0, \theta_{0} \rightarrow 0, \phi_{0} \rightarrow 0$, for $z \rightarrow \infty$

Also, $U_{1}(0)=0, N_{1}=-n\left(\frac{\partial U_{1}}{\partial z}\right)_{z=0} \theta_{1}(0)=\exp (i \omega t), \phi_{1}(0)=\exp (i \omega t)$

and $U_{1}(\infty)=0, N_{1}(\infty)=0, \theta_{1}(\infty)=0, \phi_{1}(\infty)=0$

We also use a second set of trial solutions as:

$f_{0}(z)=f_{00}(z)+E c f_{01}(z), f_{1}(z)=f_{10}(z)+E c f_{11}(z)$,

Where, the second perturbation parameter $E c$ is such that $E c<<1$ for an incompressible fluid.

Thus, using the second form of solutions in equations (3.1)-(3.10), we thus get the unperturbed parts as follows:

$(1+\xi) \frac{d^{2} U_{00}}{d z^{2}}+\frac{d U_{00}}{d z}-\frac{M^{2} \sin ^{2} \alpha}{1+m_{0}{ }^{2}}\left(1+i m_{0}\right) U_{00}=-2 \xi i \frac{d N_{00}}{d z}-G r \theta_{00}-G m \phi_{00}$

$\left(1+\frac{\xi}{2}\right) \frac{d^{2} N_{00}}{d z^{2}}+\frac{d N_{00}}{d z}-2 \xi N_{00}=0$

$\frac{1}{\operatorname{Pr}} \frac{d^{2} \theta_{00}}{d z^{2}}+\frac{d \theta_{00}}{d z}-Q_{s} \theta_{00}=0$

$\frac{1}{S c} \frac{d^{2} \phi_{00}}{d z^{2}}+\frac{d \phi_{00}}{d z}-K_{l} \phi_{00}=-S r \frac{d^{2} \theta_{00}}{d z^{2}}$

$(1+\xi) \frac{d^{2} U_{01}}{d z^{2}}+\frac{d U_{01}}{d z}-\frac{M^{2} \sin ^{2} \alpha}{1+m_{0}^{2}}\left(1+i m_{0}\right) U_{01}=-2 \xi i \frac{d N_{01}}{d z}-G r \theta_{01}-G m \phi_{01}$

$\left(1+\frac{\xi}{2}\right) \frac{d^{2} N_{01}}{d z^{2}}+\frac{d N_{01}}{d z}-2 \xi N_{01}=0$

$\frac{1}{\operatorname{Pr}} \frac{d^{2} \theta_{01}}{d z^{2}}+\frac{d \theta_{01}}{d z}-Q_{s} \theta_{01}=-\frac{M^{2} \sin ^{2} \alpha}{1+m_{0}^{2}} U_{00} \bar{U}_{00}$

$\frac{1}{S c} \frac{d^{2} \phi_{01}}{d z^{2}}+\frac{d \phi_{01}}{d z}-K_{l} \phi_{01}=-S r \frac{d^{2} \theta_{01}}{d z^{2}}$

With the boundary conditions as:

$U_{00}=u_{p}, N_{00}=-n\left(\frac{\partial U_{00}}{\partial z}\right)_{z=0} \theta_{00}=1, \phi_{00}=1$ at $z=0$

$U_{00} \rightarrow 0, N_{00} \rightarrow 0, \theta_{00} \rightarrow 0, \phi_{00} \rightarrow 0$, for $z \rightarrow \infty$

$U_{01}=0, N_{01}=-n\left(\frac{\partial U_{01}}{\partial z}\right)_{z=0} \theta_{01}=1, \phi_{01}=1$ at $z=0$

$U_{01} \rightarrow 0, N_{01} \rightarrow 0, \theta_{01} \rightarrow 0, \phi_{01} \rightarrow 0$, for $z \rightarrow \infty$ 
The perturbed parts are obtained as:

$$
(1+\xi) \frac{d^{2} U_{10}}{d z^{2}}+\delta \frac{d U_{10}}{d z}-\left(\frac{M^{2} \sin ^{2} \alpha}{1+m_{0}^{2}}\left(1+i m_{0}\right)+i \omega\right) U_{10}=-2 \xi i \frac{d N_{10}}{d z}-\delta\left(G r \theta_{00}+G m \phi_{00}\right)-\left(G r \theta_{10}+G m \phi_{10}\right)
$$

$\left(1+\frac{\xi}{2}\right) \frac{d^{2} N_{10}}{d z^{2}}+\delta \frac{d N_{10}}{d z}-(2 \xi+i \omega) N_{10}=0$

$\frac{1}{\operatorname{Pr}} \frac{d^{2} \theta_{10}}{d z^{2}}+\delta \frac{d \theta_{10}}{d z}-\left(Q_{s}+i \omega\right) \theta_{10}=0$

$\frac{1}{S c} \frac{d^{2} \phi_{10}}{d z^{2}}+\delta \frac{d \phi_{10}}{d z}-\left(K_{l}+i \omega\right) \phi_{10}=-S r \frac{d^{2} \theta_{10}}{d z^{2}}$

$(1+\xi) \frac{d^{2} U_{11}}{d z^{2}}+\delta \frac{d U_{11}}{d z}-\left(\frac{M^{2} \sin ^{2} \alpha}{1+m_{0}^{2}}\left(1+i m_{0}\right)+i \omega\right) U_{11}=-2 \xi i \frac{d N_{11}}{d z}-\delta\left(G r \theta_{01}+G m \phi_{01}\right)-\left(G r \theta_{11}+G m \phi_{11}\right)$

$\left(1+\frac{\xi}{2}\right) \frac{d^{2} N_{11}}{d z^{2}}+\delta \frac{d N_{11}}{d z}-(2 \xi+i \omega) N_{11}=0$

$\frac{1}{\operatorname{Pr}} \frac{d^{2} \theta_{11}}{d z^{2}}+\delta \frac{d \theta_{11}}{d z}-\left(Q_{s}+i \omega\right) \theta_{11}=-\frac{M^{2} \sin ^{2} \alpha}{1+m^{2}}\left(U_{00} \bar{U}_{10}+\bar{U}_{00} U_{10}\right)$

$\frac{1}{S c} \frac{d^{2} \phi_{11}}{d z^{2}}+\delta \frac{d \phi_{11}}{d z}-\left(K_{l}+i \omega\right) \phi_{11}=-S r \frac{d^{2} \theta_{11}}{d z^{2}}$

With the boundary conditions as:

$U_{10}=0, N_{10}=-n\left(\frac{\partial U_{10}}{\partial z}\right)_{z=0} \theta_{10}=1, \phi_{10}=1$ at $z=0$

and $U_{10} \rightarrow 0, N_{10} \rightarrow 0, \theta_{10} \rightarrow 0, \phi_{10} \rightarrow 0$, for $z \rightarrow \infty$

$U_{11}=0, N_{11}=-n\left(\frac{\partial U_{11}}{\partial z}\right)_{z=0} \theta_{11}=1, \phi_{11}=1$ at $z=0$

and $U_{11} \rightarrow 0, N_{11} \rightarrow 0, \theta_{11} \rightarrow 0, \phi_{11} \rightarrow 0$, for $z \rightarrow \infty$

Solving equations (1.34)-(1.51) under the boundary conditions equations (1.42)-(1.43), (1.52)-(1.53) and substituting the solutions into (1.23.1), we finally obtain,

$U(z, t)=u(z, t)+i v(z, t)$

Where $u(z, t)=u_{a}+\varepsilon\left(u_{c} \cos \omega t-u_{d} \sin \omega t\right), \quad v(z, t)=u_{b}+\varepsilon\left(u_{d} \cos \omega t+u_{c} \sin \omega t\right)$,

$u_{a}=\exp \left(-\alpha_{01} z\right)\left(c_{11} \cos \beta_{01} z+c_{12} \sin \beta_{01} z\right)+\exp \left(-m_{2} z\right) c_{3}+\exp \left(-m_{4} z\right) c_{5}+\exp \left(-m_{6} z\right) c_{7}+\exp \left(-m_{4} z\right) c_{9}$

$+E c\left(\begin{array}{l}\exp \left(-\alpha_{01} z\right)\left(r_{61} \cos \beta_{01} z+r_{62} \sin \beta_{01} z\right)+\exp \left(-m_{11} z\right) r_{1}+\exp \left(-\alpha_{12} z\right) r_{3}+\exp \left(-\alpha_{06} z\right) r_{5}+\exp \left(-\alpha_{07} z\right) r_{7}+\exp \left(-\alpha_{08} z\right) r_{9} \\ +\exp \left(-\alpha_{10} z\right) r_{11}+\exp \left(-\alpha_{02} z\right)\left(r_{13} \cos \beta_{01} z+r_{14} \sin \beta_{01} z\right)+\exp \left(-\alpha_{04} z\right)\left(r_{15} \cos \beta_{01} z+r_{16} \sin \beta_{01} z\right)+\exp \left(-\alpha_{0} z\right) \\ \left(r_{17} \cos \beta_{01} z+r_{18} \sin \beta_{01} z\right)+\exp \left(-\alpha_{02} z\right)\left(r_{19} \cos \beta_{01} z-r_{20} \sin \beta_{01} z\right)+\exp \left(-\alpha_{05} z\right) r_{21}+\exp \left(-\alpha_{04} z\right)\left(r_{23} \cos \beta_{01} z-r_{24} \sin \beta_{01} z\right) \\ +\exp \left(-m_{13} z\right) r_{29}+\exp \left(-\alpha_{11} z\right) r_{27}+\exp \left(-\alpha_{06} z\right)\left(r_{25} \cos \beta_{01} z-r_{26} \sin \beta_{01} z\right)\end{array}\right)$

$u_{b}=\exp \left(-\alpha_{01} z\right)\left(c_{12} \cos \beta_{01} z-c_{11} \sin \beta_{01} z\right)+\exp \left(-m_{2} z\right) c_{4}+\exp \left(-m_{4} z\right) c_{6}+\exp \left(-m_{6} z\right) c_{8}+\exp \left(-m_{4} z\right) c_{10}$

$+E c\left(\begin{array}{l}\exp \left(-\alpha_{01} z\right)\left(r_{62} \cos \beta_{01} z-r_{61} \sin \beta_{01} z\right)+\exp \left(-m_{11} z\right) r_{2}+\exp \left(-\alpha_{12} z\right) r_{4}+\exp \left(-\alpha_{06} z\right) r_{6}+\exp \left(-\alpha_{07} z\right) r_{7}+\exp \left(-\alpha_{08} z\right) r_{8} \\ +\exp \left(-\alpha_{10} z\right) r_{10}+\exp \left(-\alpha_{02} z\right)\left(r_{14} \cos \beta_{01} z-r_{13} \sin \beta_{01} z\right)+\exp \left(-\alpha_{04} z\right)\left(r_{16} \cos \beta_{01} z-r_{15} \sin \beta_{01} z\right)+\exp \left(-\alpha_{0} z\right) \\ \left(r_{18} \cos \beta_{01} z-r_{17} \sin \beta_{01} z\right)+\exp \left(-\alpha_{02} z\right)\left(r_{19} \cos \beta_{01} z+r_{20} \sin \beta_{01} z\right)+\exp \left(-\alpha_{05} z\right) r_{22}+\exp \left(-\alpha_{04} z\right)\left(r_{23} \cos \beta_{01} z+r_{24} \sin \beta_{01} z\right) \\ +\exp \left(-m_{13} z\right) r_{30}+\exp \left(-\alpha_{11} z\right) r_{28}+\exp \left(-\alpha_{06} z\right)\left(r_{25} \cos \beta_{01} z+r_{26} \sin \beta_{01} z\right)\end{array}\right)$ 
$u_{c}=\exp \left(-\alpha_{01} z\right)\left(d_{24} \cos \beta_{01} z+d_{25} \sin \beta_{01} z\right)+\exp \left(-\alpha_{02} z\right)\left(d_{10} \cos \beta_{02} z+d_{11} \sin \beta_{02} z\right)+\exp \left(-\alpha_{14} z\right)\left(d_{20} \cos \beta_{02} z+d_{21} \sin \beta_{02} z\right)$ $+\exp \left(-\alpha_{03} z\right)\left(d_{18} \cos \beta_{03} z+d_{19} \sin \beta_{03} z\right)+\exp \left(-m_{4} z\right) d_{12}+\exp \left(-m_{6} z\right) d_{14}+\exp \left(-m_{4} z\right) d_{16}$

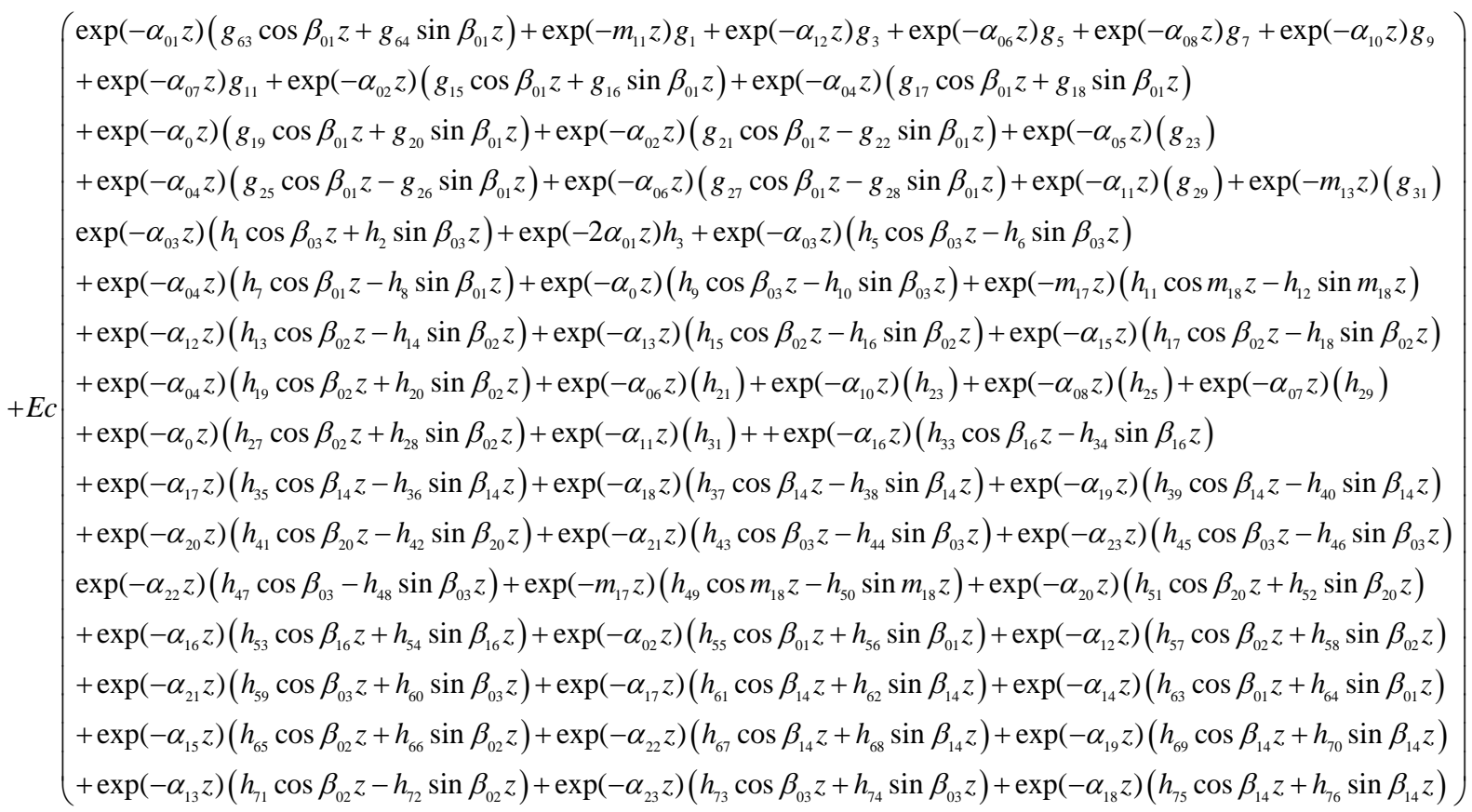

$u_{d}=\exp \left(-\alpha_{01} z\right)\left(d_{25} \cos \beta_{01} z-d_{24} \sin \beta_{01} z\right)+\exp \left(-\alpha_{02} z\right)\left(d_{11} \cos \beta_{02} z-d_{10} \sin \beta_{02} z\right)+\exp \left(-\alpha_{14} z\right)\left(d_{21} \cos \beta_{02} z-d_{20} \sin \beta_{02} z\right)$ $+\exp \left(-\alpha_{03} z\right)\left(d_{19} \cos \beta_{03} z-d_{20} \sin \beta_{03} z\right)+\exp \left(-m_{4} z\right) d_{13}+\exp \left(-m_{6} z\right) d_{15}+\exp \left(-m_{4} z\right) d_{17}$

$\left(\exp \left(-\alpha_{01} z\right)\left(g_{64} \cos \beta_{01} z-g_{63} \sin \beta_{01} z\right)+\exp \left(-m_{11} z\right) g_{2}+\exp \left(-\alpha_{12} z\right) g_{4}+\exp \left(-\alpha_{06} z\right) g_{6}+\exp \left(-\alpha_{08} z\right) g_{87}\right.$

$+\exp \left(-\alpha_{10} z\right) g_{10}+\exp \left(-\alpha_{07} z\right) g_{12}+\exp \left(-\alpha_{02} z\right)\left(g_{16} \cos \beta_{01} z+g_{15} \sin \beta_{01} z\right)+\exp \left(-\alpha_{04} z\right)\left(g_{18} \cos \beta_{01} z-g_{17} \sin \beta_{01} z\right)$

$+\exp \left(-\alpha_{0} z\right)\left(g_{20} \cos \beta_{01} z-g_{19} \sin \beta_{01} z\right)+\exp \left(-\alpha_{02} z\right)\left(g_{22} \cos \beta_{01} z+g_{21} \sin \beta_{01} z\right)+\exp \left(-\alpha_{05} z\right)\left(g_{24}\right)$

$+\exp \left(-\alpha_{04} z\right)\left(g_{26} \cos \beta_{01} z+g_{25} \sin \beta_{01} z\right)+\exp \left(-\alpha_{06} z\right)\left(g_{28} \cos \beta_{01} z+g_{27} \sin \beta_{01} z\right)+\exp \left(-\alpha_{11} z\right)\left(g_{30}\right)$

$+\exp \left(-m_{13} z\right)\left(g_{32}\right) \exp \left(-\alpha_{03} z\right)\left(h_{2} \cos \beta_{03} z-h_{1} \sin \beta_{03} z\right)+\exp \left(-2 \alpha_{01} z\right) h_{4}+\exp \left(-\alpha_{03} z\right)\left(h_{6} \cos \beta_{03} z+h_{5} \sin \beta_{03} z\right)$

$+\exp \left(-\alpha_{04} z\right)\left(h_{8} \cos \beta_{01} z+h_{7} \sin \beta_{01} z\right)+\exp \left(-\alpha_{0} z\right)\left(h_{10} \cos \beta_{03} z+h_{9} \sin \beta_{03} z\right)+\exp \left(-m_{17} z\right)\left(h_{12} \cos m_{18} z-h_{11} \sin m_{18} z\right)$

$+\exp \left(-\alpha_{12} z\right)\left(h_{14} \cos \beta_{02} z+h_{13} \sin \beta_{02} z\right)+\exp \left(-\alpha_{13} z\right)\left(h_{16} \cos \beta_{02} z+h_{15} \sin \beta_{02} z\right)+\exp \left(-\alpha_{15} z\right)\left(h_{18} \cos \beta_{02} z+h_{17} \sin \beta_{02} z\right)$

$+E c$

$+\exp \left(-\alpha_{04} z\right)\left(h_{20} \cos \beta_{02} z-h_{19} \sin \beta_{02} z\right)+\exp \left(-\alpha_{06} z\right)\left(h_{22}\right)+\exp \left(-\alpha_{10} z\right)\left(h_{24}\right)+\exp \left(-\alpha_{08} z\right)\left(h_{26}\right)+\exp \left(-\alpha_{07} z\right)\left(h_{30}\right)$

$+\exp \left(-\alpha_{0} z\right)\left(h_{28} \cos \beta_{02} z-h_{27} \sin \beta_{02} z\right)+\exp \left(-\alpha_{11} z\right)\left(h_{32}\right)++\exp \left(-\alpha_{16} z\right)\left(h_{34} \cos \beta_{16} z+h_{33} \sin \beta_{16} z\right)$

$+\exp \left(-\alpha_{17} z\right)\left(h_{36} \cos \beta_{14} z+h_{35} \sin \beta_{14} z\right)+\exp \left(-\alpha_{18} z\right)\left(h_{38} \cos \beta_{14} z+h_{37} \sin \beta_{14} z\right)+\exp \left(-\alpha_{19} z\right)\left(h_{40} \cos \beta_{14} z+h_{39} \sin \beta_{14} z\right)$

$+\exp \left(-\alpha_{20} z\right)\left(h_{42} \cos \beta_{20} z+h_{41} \sin \beta_{20} z\right)+\exp \left(-\alpha_{21} z\right)\left(h_{44} \cos \beta_{03} z+h_{43} \sin \beta_{03} z\right)+\exp \left(-\alpha_{23} z\right)\left(h_{46} \cos \beta_{03} z+h_{45} \sin \beta_{03} z\right)$

$+\exp \left(-\alpha_{22} z\right)\left(h_{48} \cos \beta_{03}+h_{47} \sin \beta_{03} z\right)+\exp \left(-m_{17} z\right)\left(h_{50} \cos m_{18} z+h_{49} \sin m_{18} z\right)+\exp \left(-\alpha_{20} z\right)\left(h_{52} \cos \beta_{20} z-h_{51} \sin \beta_{20} z\right)$

$+\exp \left(-\alpha_{16} z\right)\left(h_{54} \cos \beta_{16}-h_{53} \sin \beta_{16} z\right)+\exp \left(-\alpha_{02} z\right)\left(h_{56} \cos \beta_{01} z-h_{55} \sin \beta_{01} z\right)+\exp \left(-\alpha_{12} z\right)\left(h_{58} \cos \beta_{02} z-h_{57} \sin \beta_{02} z\right)$

$+\exp \left(-\alpha_{21} z\right)\left(h_{60} \cos \beta_{03} z-h_{59} \sin \beta_{03} z\right)+\exp \left(-\alpha_{17} z\right)\left(h_{62} \cos \beta_{14} z-h_{61} \sin \beta_{14} z\right)+\exp \left(-\alpha_{14} z\right)\left(h_{64} \cos \beta_{01} z-h_{63} \sin \beta_{01} z\right)$

$+\exp \left(-\alpha_{15} z\right)\left(h_{66} \cos \beta_{02} z-h_{65} \sin \beta_{02} z\right)+\exp \left(-\alpha_{22} z\right)\left(h_{68} \cos \beta_{14} z-h_{67} \sin \beta_{14} z\right)+\exp \left(-\alpha_{19} z\right)\left(h_{70} \cos \beta_{14} z-h_{69} \sin \beta_{14} z\right)$

$\left.+\exp \left(-\alpha_{13} z\right)\left(h_{72} \cos \beta_{02} z-h_{71} \sin \beta_{02} z\right)+\exp \left(-\alpha_{23} z\right)\left(h_{74} \cos \beta_{03} z-h_{73} \sin \beta_{03} z\right)+\exp \left(-\alpha_{18} z\right)\left(h_{76} \cos \beta_{14} z-h_{75} \sin \beta_{14} z\right)\right)$

$\phi_{R}(z, t)=\phi_{0 R}(z, t)+\varepsilon\left(\cos (\omega t) \phi_{11 a}-\sin (\omega t) \phi_{11 b}\right)=\left(\phi_{00}(R)+E c \phi_{01}(R)\right)+\varepsilon\left(\cos (\omega t) \phi_{11 a}-\sin (\omega t) \phi_{11 b}\right)$

Where

$$
\phi_{00 R}=\exp \left(-m_{6} z\right) c_{4}+c_{3} \exp \left(-m_{4} z\right)
$$




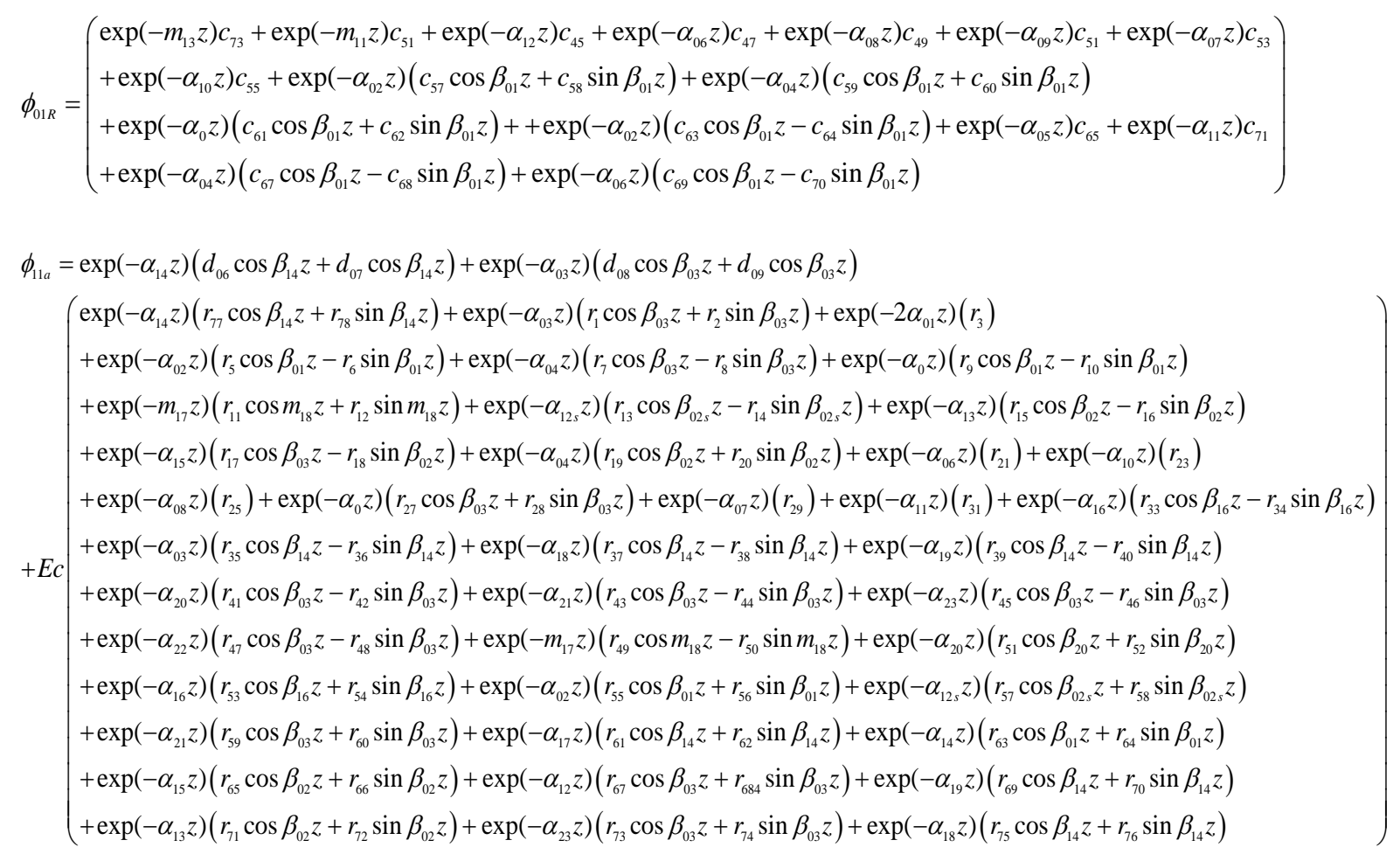

$\phi_{11 b}=\exp \left(-\alpha_{14} z\right)\left(d_{07} \cos \beta_{14} z-d_{06} \cos \beta_{14} z\right)+\exp \left(-\alpha_{03} z\right)\left(d_{09} \cos \beta_{03} z-d_{08} \cos \beta_{03} z\right)$

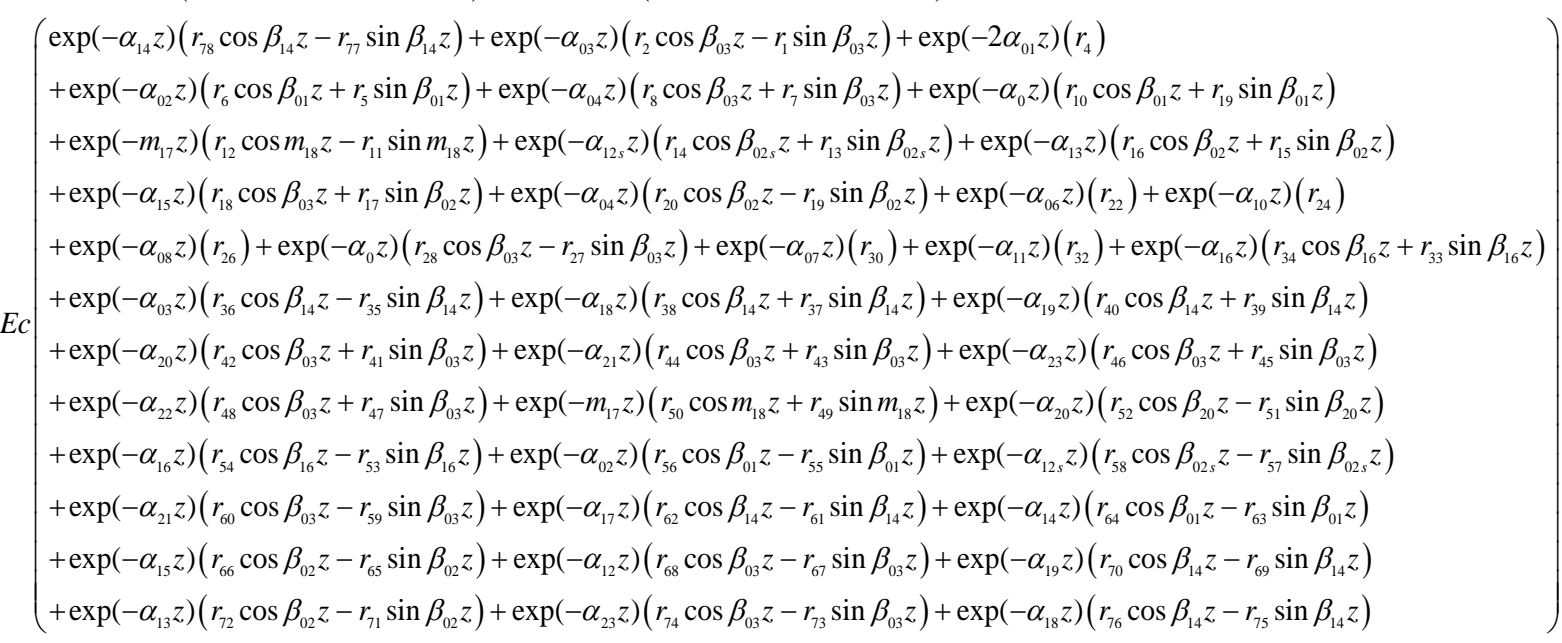

$\theta_{R}(z, t)=\theta_{0 R}(z, t)+\varepsilon\left(\cos (\omega t) \theta_{11 a}-\sin (\omega t) \theta_{11 b}\right)=\left(\theta_{00 R}(z, t)+E c \theta_{01 R}(z, t)\right)+\varepsilon\left(\cos (\omega t) \theta_{11 a}-\sin (\omega t) \theta_{11 b}\right)$

\section{Where}

$\theta_{00 R}=\exp \left(-m_{4} z\right)$

$\theta_{01 R}=\left(\begin{array}{l}\exp \left(-m_{11} z\right)\left(c_{43}\right)+\exp \left(-\alpha_{12} z\right)\left(c_{13}\right)+\exp \left(-\alpha_{06} z\right)\left(c_{15}\right)+\exp \left(-\alpha_{08} z\right)\left(c_{17}\right)+\exp \left(-\alpha_{09} z\right)\left(c_{19}\right)+\exp \left(-\alpha_{07} z\right)\left(c_{21}\right)+\exp \left(-\alpha_{10} z\right)\left(c_{23}\right) \\ +\exp \left(-\alpha_{02} z\right)\left(c_{25} \cos \beta_{1} z+c_{26} \sin \beta_{1} z\right)+\exp \left(-\alpha_{04} z\right)\left(c_{27} \cos \beta_{2} z+c_{28} \sin \beta_{2} z\right)+\exp \left(-\alpha_{0} z\right)\left(c_{29} \cos \beta_{01} z+c_{30} \sin \beta_{01} z\right)+\exp \left(-\alpha_{11} z\right)\left(c_{41}\right) \\ +\exp \left(-\alpha_{02} z\right)\left(c_{31} \cos \beta_{2} z-c_{32} \sin \beta_{2} z\right)+\exp \left(-\alpha_{05} z\right)\left(c_{33}\right)+\exp \left(-\alpha_{04} z\right)\left(c_{35} \cos \beta_{01} z-c_{36} \sin \beta_{01} z\right)+\exp \left(-\alpha_{06} z\right)\left(c_{37} \cos \beta_{01} z-c_{38} \sin \beta_{01} z\right)\end{array}\right)$ 

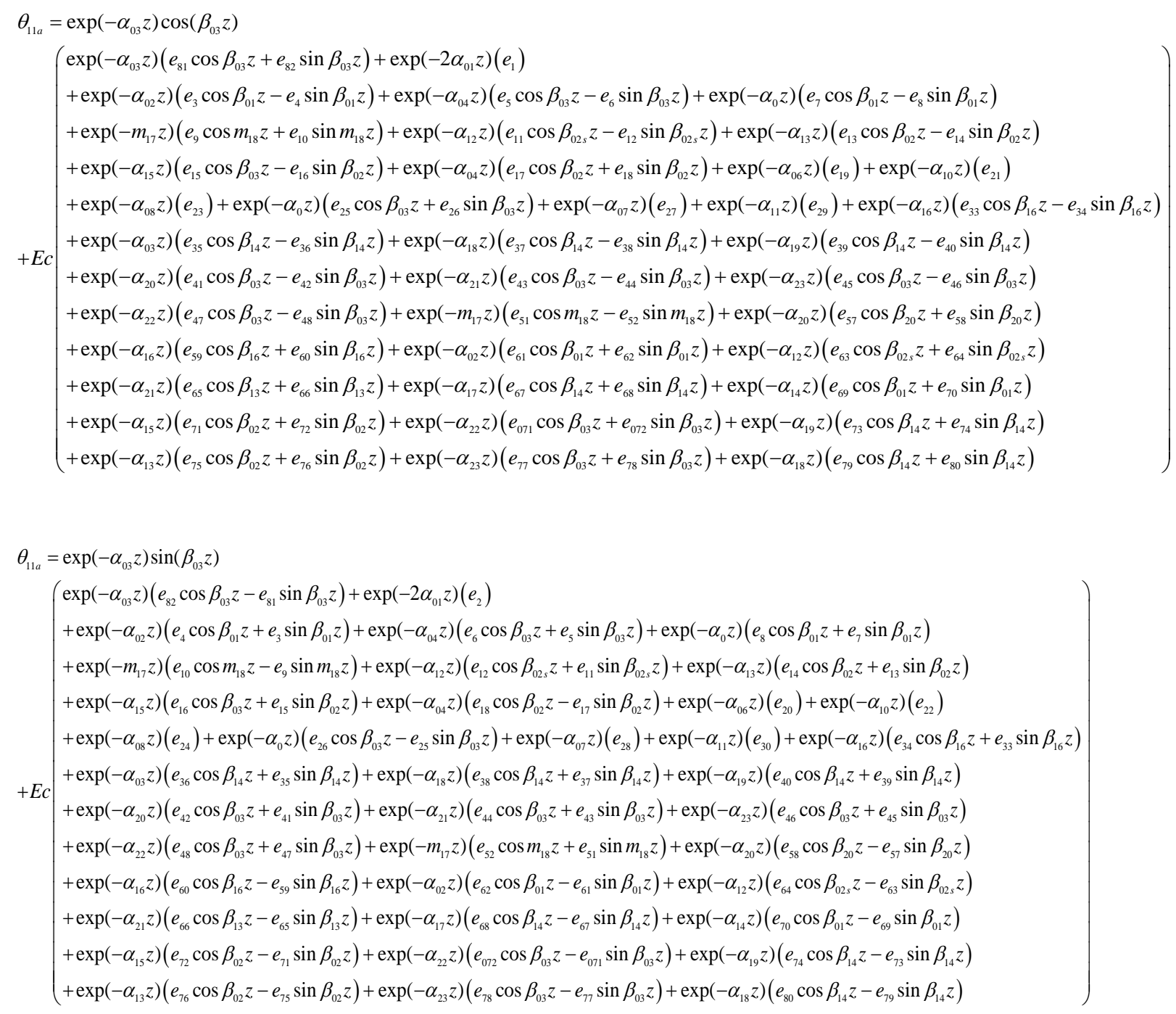

Some quantities of engineering interest are discussed as follows:

\subsection{Nusselt number}

The non-dimensional heat transfer rate at the plate $z=0$ is given by:

$$
N u_{R}=-\frac{1}{\operatorname{Pr}}\left(\frac{\partial \theta_{R}}{\partial z}\right)_{z=0}=-\frac{1}{\operatorname{Pr}}\left(N u_{01}+N u_{02}+N u_{03}\right)
$$

\section{Where}

$$
\left.\begin{array}{l}
N u_{01}=-m_{4}+E c\left(\begin{array}{l}
-m_{11} c_{43}-\alpha_{12} c_{13}-\alpha_{06} c_{15}-\alpha_{08} c_{17}-\alpha_{09} c_{19}-\alpha_{07} c_{21}-\alpha_{10} c_{23}-\alpha_{02} c_{25}+c_{26} \beta-\alpha_{04} c_{27} \\
+\beta_{2} c_{28}-\alpha_{0} c_{29}+c_{30} \beta_{01}-\alpha_{02} c_{31}-c_{32} \beta_{2}-\alpha_{05} c_{33}-\alpha_{04} c_{35}-\beta_{01} c_{36}-\alpha_{06} c_{37}-\beta_{01} c_{38}-\alpha_{11} c_{41}
\end{array}\right) \\
\left.N u_{02}=\varepsilon \cos \omega t\left(\begin{array}{l}
-\alpha_{03} e_{81}+e_{82} \beta_{03}-2 \alpha_{01} e_{1}-\alpha_{02} e_{3}-\beta_{01} e_{4}-\alpha_{01} e_{5}-\beta_{03} e_{6}-\alpha_{0} e_{7}-\beta_{01} e_{8}-m_{17} e_{9}+e_{10} m_{18}-e_{11} \alpha_{12}^{*}-e_{12} \beta_{02}^{*}-e_{13} \alpha_{13}-e_{14} \beta_{02}^{*}-e_{15} \alpha_{15} \\
-e_{16} \beta_{02}^{*}-\alpha_{04} e_{17}+e_{18} \beta_{02}-\alpha_{06} e_{19}-\alpha_{10} e_{21}-\alpha_{08} e_{23}-\alpha_{0} e_{25}+e_{26} \beta_{03}-\alpha_{07} e_{27}-\alpha_{11} e_{29}-\alpha_{16} e_{33}-\beta_{16} e_{34}-\alpha_{03} e_{35}-e_{36} \beta_{14}-\alpha_{19} e_{39}-\beta_{14} e_{40} \\
-\alpha_{20} e_{41}-e_{42} \beta_{03}-\alpha_{21} e_{43}-e_{44} \beta_{03}-\alpha_{23} e_{45}-\beta_{03} e_{46}-\alpha_{22} e_{47}-e_{48} \beta_{03}-m_{17} e_{51}-e_{52} m_{18}-\alpha_{20} e_{57}+e_{58} \beta_{20}-\alpha_{16} e_{59}+e_{60} \beta_{16}-\alpha_{02} e_{61}+e_{62} \beta_{01} \\
-\alpha_{12} e_{63}+e_{64} \beta_{02}^{*}-e_{65} \alpha_{21}+e_{66} \beta_{13}-e_{67} \alpha_{17}+e_{68} \beta_{14}-\alpha_{14} e_{69}+e_{70} \beta_{01}-\alpha_{15} e_{71}+e_{72} \beta_{02}-\alpha_{22} e_{071}+e_{072} \beta_{03}-\alpha_{19} e_{73}+e_{74} \beta_{14}-\alpha_{13} e_{75}+e_{76} \beta_{02} \\
+\beta_{03} e_{78}-e_{77} \alpha_{23}+\beta_{14} e_{80}-e_{79} \alpha_{18}
\end{array}\right)\right)
\end{array}\right)
$$


$N u_{02}=-\varepsilon \sin \omega t\left(\beta_{03}+E c\left(\begin{array}{l}-\alpha_{03} e_{82}-e_{81} \beta_{03}-2 \alpha_{01} e_{2}-\alpha_{02} e_{4}+\beta_{01} e_{3}-\alpha_{04} e_{6}+\beta_{03} e_{5}-\alpha_{0} e_{8}+\beta_{01} e_{7}-m_{17} e_{10}-e_{9} m_{18}-e_{12} \alpha_{12}^{*}+e_{11} \beta_{02}^{*}-e_{14} \alpha_{13}+e_{13} \beta_{02}^{*}-e_{16} \alpha_{15} \\ +e_{15} \beta_{02}^{*}-\alpha_{04} e_{18}-e_{17} \beta_{02}-\alpha_{06} e_{20}-\alpha_{10} e_{22}-\alpha_{08} e_{24}-\alpha_{0} e_{26}-e_{25} \beta_{03}-\alpha_{07} e_{28}-\alpha_{11} e_{30}-\alpha_{16} e_{34}+\beta_{16} e_{33}-\alpha_{03} e_{36}+e_{35} \beta_{14}-\alpha_{18} e_{38}+\beta_{14} e_{37} \\ -\alpha_{19} e_{40}+e_{39} \beta_{14}-\alpha_{20} e_{42}+e_{41} \beta_{03}-\alpha_{21} e_{44}+\beta_{03} e_{43}-\alpha_{23} e_{46}+e_{45} \beta_{03}-\alpha_{22} e_{48}-e_{52} m_{17}-\alpha_{20} e_{58}+e_{51} m_{18}-\beta_{20} e_{57}-e_{60} \alpha_{16}-\beta_{16} e_{59}-e_{62} \alpha_{02} \\ -e_{61} \beta_{01}-\alpha_{12} e_{64}-e_{63} \beta_{02}^{*}-\alpha_{21} e_{66}-e_{65} \beta_{13}-\alpha_{17} e_{68}-\beta_{14} e_{67}-\alpha_{14} e_{70}-e_{69} \beta_{01}-\alpha_{15} e_{72}-\alpha_{71} \beta_{02}-\alpha_{22} e_{072}-\alpha_{19} e_{74}-\beta_{14} e_{73}-\alpha_{13} e_{76}-e_{75} \beta_{02} \\ -\alpha_{23} e_{78}-e_{77} \beta_{03}-e_{80} \alpha_{18}-e_{79} \beta_{14}\end{array}\right)\right)$

\subsection{Sherwood number}

The non-dimensional mass transfer rate at the plate $z=0$ is found as:

$$
S h_{R}=-\frac{1}{S c}\left(\frac{\partial \phi_{R}}{\partial z}\right)_{z=0}=-\frac{1}{S c}\left(S h_{01}+S h_{\mathrm{O} 2}+S h_{\mathrm{O} 3}\right)
$$

Where

$S h_{01}=\left(-m_{6} c_{4}-m_{4} c_{3}\right)+E c\left(\begin{array}{l}-m_{13} c_{73}-m_{11} c_{51}-\alpha_{12} c_{45}-\alpha_{06} c_{47}-\alpha_{08} c_{49}-\alpha_{09} c_{51}-\alpha_{07} c_{53}-\alpha_{10} c_{55}-\alpha_{02} c_{57}+c_{58} \beta_{01}-\alpha_{04} c_{59} \\ +c_{60} \beta_{01}-\alpha_{0} c_{61}+c_{62} \beta_{01}-\alpha_{02} c_{63}-c_{64} \beta_{01}-\alpha_{05} c_{65}-\alpha_{11} c_{71}-\alpha_{04} c_{67}-c_{68} \beta_{01}-\alpha_{06} c_{69}-c_{70} \beta_{01}\end{array}\right)$

$S h_{02}=\varepsilon \cos \omega t\left(-\alpha_{14} d_{06}+d_{07} \beta_{14}--\alpha_{03} d_{08}+d_{09} \beta_{03}\right)$

$\left.+\varepsilon \cos \omega t\left(\begin{array}{l}-\alpha_{14} r_{77}+r_{78} \beta_{14}-\alpha_{03} r_{1}+r_{2} \beta_{03}-2 \alpha_{01} r_{3}-\alpha_{02} r_{5}-r_{6} \beta_{01}-\alpha_{04} r_{7}-r_{8} \beta_{03}-\alpha_{0} r_{9}-r_{10} \beta_{01}-m_{17} r_{11}+r_{12} m_{18}-\alpha_{12}^{*} r_{13}-r_{14} \beta_{02}^{*}-\alpha_{13} r_{15}-r_{16} \beta_{02} \\ -\alpha_{15} r_{17}-r_{18} \beta_{02}-\alpha_{04} r_{19}+r_{20} \beta_{02}-\alpha_{06} r_{21}-\alpha_{10} r_{23}-\alpha_{08} r_{25}-\alpha_{0} r_{27}+r_{28} \beta_{03}-\alpha_{07} r_{29}-\alpha_{11} r_{31}-\alpha_{16} r_{33}-r_{34} \beta_{16}-\alpha_{03} r_{35}-r_{36} \beta_{14}-\alpha_{18} r_{37}-r_{38} \beta_{14} \\ -\alpha_{19} r_{39}-r_{40} \beta_{14}-\alpha_{20} r_{41}-r_{42} \beta_{03}-\alpha_{21} r_{43}-r_{44} \beta_{03}-\alpha_{23} r_{45}-r_{46} \beta_{03}-\alpha_{22} r_{47}-r_{48} \beta_{03}-m_{17} r_{49}-r_{50} m_{18}-\alpha_{20} r_{51}+r_{52} \beta_{20}-\alpha_{16} r_{53}+r_{54} \beta_{16}-\alpha_{02} r_{55} \\ +r_{56} \beta_{01}-\alpha_{12}^{*} r_{57}+r_{58} \beta_{02}^{*}-\alpha_{21} r_{59}+r_{60} \beta_{03}-\alpha_{17} r_{61}+r_{62} \beta_{14}-\alpha_{14} r_{63}+r_{64} \beta_{01}-\alpha_{15} r_{65}+r_{66} \beta_{02}-\alpha_{12} r_{67}+r_{68} \beta_{03}-\alpha_{19} r_{69}+r_{70} \beta_{14}-\alpha_{13} r_{71}+r_{72} \beta_{02} \\ -\alpha_{23} r_{73}+r_{74} \beta_{03}-\alpha_{18} r_{75}+r_{76} \beta_{14}\end{array}\right)\right)$

$S h_{03}=\varepsilon \sin \omega t\left[E c\left(\begin{array}{l}-\alpha_{19} r_{39}-\beta_{14} r_{40}-\alpha_{20} r_{41}-\beta_{03} r_{42}-\alpha_{21} r_{43}-\beta_{03} r_{44}-r_{45} \alpha_{23}-\beta_{03} r_{46}-r_{47} \alpha_{22}-\beta_{03} r_{48}-r_{49} m_{17}-m_{18} r_{50}-\alpha_{20} r_{51} \\ +\beta_{20} r_{52}-\alpha_{16} r_{53}+r_{54} \beta_{16}-\alpha_{02} r_{55}+\beta_{01} r_{56}-\alpha_{12}^{*} r_{57}+\beta_{02} r_{58}-\alpha_{21} r_{59}+\beta_{03} r_{60}-\alpha_{17} r_{61}+\beta_{14} r_{62}-\alpha_{14} r_{63}+\beta_{01} r_{64} \\ -\alpha_{15} r_{65}+\beta_{02} r_{66}-\alpha_{12} r_{67}+\beta_{03} r_{68}-\alpha_{19} r_{69}+\beta_{14} r_{70}-\alpha_{13} r_{71}+\beta_{02} r_{72}-\alpha_{23} r_{73}+\beta_{03} r_{74}-\alpha_{18} r_{75}+\beta_{14} r_{76}\end{array}\right)\right]$

\subsection{Skin friction coefficient}

$$
\begin{aligned}
& C f_{R}=2(1+\xi(1-n))\left(\frac{\partial U_{R}}{\partial z}\right)_{z=0} \\
& =2(1+\xi(1-n))\left(c f_{1}+c f_{2}+c f_{3}\right)
\end{aligned}
$$

Where

$$
\begin{aligned}
& c f_{1}=\left(\begin{array}{l}
-\alpha_{0} c_{1}+\beta_{01} c_{12}-m_{2} c_{3}-m_{4} c_{5}-m_{6} c_{7}-m_{4} c_{9}-\alpha_{01} r_{61}+\beta_{01} r_{62}-m_{11} r_{1}-\alpha_{12} r_{3}-\alpha_{06} r_{5}-\alpha_{07} r_{7}-\alpha_{09} r_{9}-\alpha_{10} r_{11}-\alpha_{02} r_{13} \\
+\beta_{01} r_{14}-\alpha_{04} r_{15}+\beta_{01} r_{16}-\alpha_{0} r_{17}+\beta_{01} r_{18}-\alpha_{02} r_{19}+\beta_{01} r_{20}-\alpha_{05} r_{21}-\alpha_{04} r_{23}+r_{24} \beta_{01}-\alpha_{0} r_{25}+\beta_{01} r_{26}-\alpha_{11} r_{27}-m_{13} r_{29}
\end{array}\right), \\
& c f_{2}=\cos \omega t\left(\begin{array}{l}
-\alpha_{01} d_{24}+\beta_{01} d_{25}-\alpha_{025} d_{10}+d_{11} \beta_{025}-m_{4} d_{12}-m_{6} d_{14}-m_{4} d_{16}-\alpha_{03} d_{18}+d_{19} \beta_{03}-\alpha_{14} d_{20}+d_{21} \beta_{14} \\
-\alpha_{01} g_{63}+\beta_{01} g_{64}-m_{11} g_{1}-\alpha_{12} g_{3}-\alpha_{06} g_{7}-\alpha_{10} g_{9}-\alpha_{07} g_{11}-\alpha_{02} g_{15}-\alpha_{04} g_{17}-\alpha_{0} g_{19}-\alpha_{02} g_{21} \\
-\beta_{01} g_{22}-\alpha_{03} g_{23}-\alpha_{04} g_{25}-\beta_{01} g_{26}-\alpha_{0} g_{27}-\beta_{01} g_{28}-\alpha_{11} g_{29}-g_{31} m_{13}-h_{1} \alpha_{03}+h_{2} \beta_{03}-2 \alpha_{01} h_{3} \\
-\alpha_{02} h_{5}-h_{6} \beta_{01}-\alpha_{04} h_{7}-\beta_{01} h_{8}-\alpha_{0} h_{9}-\beta_{01} h_{10}-m_{17} h_{11}+h_{12} m_{18}-h_{13} \alpha_{12}^{*}-h_{14} \beta_{12}^{*}-\alpha_{13} h_{15}-h_{16} \beta_{02}^{*} \\
-\alpha_{15} h_{17}-h_{18} \beta_{02}^{*}-\alpha_{04} h_{19}+h_{20} \beta_{02}-\alpha_{06} h_{21}-\alpha_{10} h_{23}-\alpha_{08} h_{25}-\alpha_{0} h_{27}-\alpha_{07} h_{29}-\alpha_{11} h_{31}-\alpha_{16} h_{33} \\
-h_{34} \beta_{16}-\alpha_{17} h_{35}-h_{36} \beta_{14}-\alpha_{18} h_{37}-h_{38} \beta_{14}-h_{39} \alpha_{19}-h_{40} \beta_{14}-h_{41} \alpha_{20}-h_{36} \beta_{20}-\alpha_{18} h_{37}-h_{38} \beta_{14} \\
-h_{39} \alpha_{19}-h_{40} \beta_{14}-\alpha_{20} h_{41}-h_{42} \beta_{20}-\alpha_{21} h_{43}-h_{44} \beta_{03}-\alpha_{23} h_{45}-h_{46} \beta_{03}-h_{47} \alpha_{22}-h_{48} \beta_{03}-h_{49} m_{17} \\
-h_{50} m_{18}-h_{51} \alpha_{50}+h_{52} \beta_{20}-h_{53} \alpha_{16}+h_{54} \beta_{16}-h_{55} \alpha_{02}+h_{56} \beta_{01}-h_{57} \alpha_{12}^{*}+h_{58} \alpha_{02}^{*}-h_{59} \alpha_{21}+\beta_{03} h_{60} \\
-h_{61} \alpha_{17}+h_{62} \beta_{14}-h_{63} \alpha_{14}+h_{64} \beta_{01}-h_{65} \alpha_{15}+h_{66} \beta_{02}-h_{67} \alpha_{22}-h_{69} \alpha_{19}-h_{71} \alpha_{13}-h_{73} \alpha_{23}-h_{75} \alpha_{18} \\
+h_{68} \beta_{03}+h_{70} \beta_{14}+h_{72} \beta_{02}^{*}+h_{74} \beta_{03}+h_{76} \beta_{14}
\end{array}\right),
\end{aligned}
$$




$$
c f_{3}=-\sin \omega t\left(\begin{array}{l}
-\alpha_{01} d_{25}+\beta_{01} d_{24}-\alpha_{02}^{*} d_{11}-\beta_{02}^{*} d_{10}-m_{4} d_{13}-m_{6} d_{15}-m_{4} d_{17}-\alpha_{03} d_{19}-d_{18} \beta_{03}-\alpha_{14} d_{21}-\beta_{14} d_{20}-\alpha_{01} g_{64}-\beta_{01} g_{63} \\
-m_{11} g_{2}-\alpha_{12} g_{4}-g_{6} \alpha_{06}-\alpha_{08} g_{8}-\alpha_{10} g_{10}-\alpha_{07} g_{12}-\alpha_{02} g_{16}-\beta_{01} g_{15}-\alpha_{04} g_{16}-\beta_{01} g_{17}-\alpha_{0} g_{20}-\beta_{01} g_{19}-\alpha_{02} g_{22} \\
+\beta_{01} g_{21}-\alpha_{03} g_{24}-\alpha_{04} g_{26}+\beta_{01} g_{25}-g_{28} \alpha_{0}+\beta_{01} g_{27}-\alpha_{11} g_{30}-m_{13} g_{32}-\alpha_{03} h_{2}-\beta_{03} h_{1}-2 \alpha_{01} h_{4}-\alpha_{02} h_{6}+\beta_{01} h_{5} \\
-\alpha_{04} h_{8}+\beta_{01} h_{7}-\alpha_{0} h_{10}+\beta_{01} h_{9}-h_{12} m_{17}+h_{11} m_{18}+\beta_{02}^{*} h_{13}-\alpha_{12}^{*} h_{14}-\alpha_{13} h_{16}+\beta_{02}^{*} h_{15}-\alpha_{15} h_{18}+\beta_{02}^{*} h_{17}-\alpha_{04} h_{20} \\
-\beta_{02} h_{19}-\alpha_{06} h_{22}-\alpha_{10} h_{24}-\alpha_{08} h_{26}-h_{28} \alpha_{0}-\beta_{01} h_{27}-h_{30} \alpha_{07}-\alpha_{11} h_{32}+\beta_{18} h_{33}-\alpha_{10} h_{34}+\beta_{14} h_{35}-h_{36} \alpha_{17}+\beta_{14} h_{37} \\
-\alpha_{18} h_{38}+\beta_{14} h_{39}-\alpha_{19} h_{40}-\alpha_{20} h_{42}+\beta_{20} h_{41}-\alpha_{21} h_{44}+\beta_{03} h_{43}-\alpha_{23} h_{46}+\beta_{03} h_{45}-\alpha_{22} h_{48}+\beta_{03} h_{47}-m_{17} h_{50}+m_{18} h_{49} \\
-\alpha_{10} h_{52}-\beta_{20} h_{51}-\alpha_{16} h_{54}-h_{53} \beta_{16}-\alpha_{02} h_{56}-\beta_{01} h_{55}-\alpha_{12}^{*} h_{58}-\beta_{02}^{*} h_{57}-\beta_{03} h_{59}-\alpha_{21} h_{60}-\beta_{14} h_{61}-\alpha_{17} h_{62}-\beta_{01} h_{63} \\
-\alpha_{14} h_{64}-\alpha_{15} h_{66}-\beta_{03} h_{68}-\alpha_{22} h_{67}-\beta_{14} h_{69}-\alpha_{19} h_{70}-\beta_{02}^{*} h_{71}-\alpha_{13} h_{72}-\beta_{03} h_{74}-\alpha_{23} h_{73}-\beta_{14} h_{75}-\alpha_{18} h_{76}
\end{array}\right)-
$$

\subsection{Couple stress coefficient}

$$
\begin{aligned}
& C m_{R}=\left(1+\frac{1}{2} \xi\right) N_{R}^{\prime}(\mathrm{O}) \\
& =-\left(c_{1} m_{2}\right)+\varepsilon\left(\cos \omega t\left(-\alpha_{02} d_{04}+d_{05} \beta_{02}\right)+\sin \omega t\left(\alpha_{02} d_{05}+d_{04} \beta_{02}\right)\right)
\end{aligned}
$$

\section{Numerical Data Analysis}

A numerical estimation is carried out to comprehend the substantial significance of the flow problem of the three dimensional unsteady MHD micropolar fluid with Hall effect, Joule heating and heat sink in the presence of gravitational modulation. The governing non-dimensional equations are solved with the help of perturbation technique. The results are analyzed with the help of graphs and tables. The non-dimensional velocity, temperature and concentration profiles are discussed numerically. The skin friction and couple stress coefficient in addition to the rate of heat and mass transfers are also computed numerically. In the present problem, the value of Prandtl number $(\mathrm{Pr})$ is taken as 7.0 which relates to water $\left(\mathrm{H}_{2} \mathrm{O}\right)$ at $25^{\circ} \mathrm{C}$ and $\mathrm{Schmidt}$ number is taken as 0.60 which relates to water vapour at $20^{\circ} \mathrm{C}$. Prandtl number is a dimensionless physical parameter, which is the ratio between momentum diffusivity and thermal diffusivity. In mathematical strategy, Prandtl number is expressed as, $\operatorname{Pr}=\frac{v}{\alpha_{o}}$ where $v=\frac{\mu_{0}}{\rho}, \alpha_{0}=\frac{k}{\rho C_{p}}$ and $v$ is the momentum diffusivity, $\mu_{0}, \alpha_{0}, k, C p, \rho$ denote the dynamic viscosity, thermal diffusivity, thermal conductivity, specific heat and density respectively. The Prandtl number is an estimation of comparative persuasion of momentum and heat energy in a fluid flow. For $\operatorname{Pr}<<1$ i.e. for small values of Prandtl number, the thermal diffusivity dominates where as for large values of Prandtl number, i.e for $\operatorname{Pr} \gg>1$, the momentum diffusivity dominates. The value of Prandtl number for water at temperature $25^{0} \mathrm{C}, k=0.607 \mathrm{Wm}^{-1} \mathrm{~K}^{-1}, \mu_{0}=1.002 \mathrm{mPas}, C_{P} \square 4.182 \mathrm{JKg}^{-1} \mathrm{~K}^{-1}$ is approximately found as 7.0. In this regard, convection is more effective in transferring energy compare to conduction i.e. the momentum diffusivity is more dominant. Since the momentum diffusivity will dominate in micropolar fluid, so in this case, the value of Prandtl number i.e $P r>>1$.This is why, the value of Prandtl number $(P r)$ is assumed as 7.0 in this problem. The Schmidt number is also a dimensionless parameter, which is a measure of momentum diffusivity relative to mass diffusivity. Mathematically, $S c=\frac{v}{D_{M}}$ where $D_{M}$ is the mass diffusivity of species and $v$ is the kinematic viscosity. Schmidt number is employed to trace the fluid motion in which both momentum and mass diffusion occurs together. To justify the fluid modeling the values of other flow variables are taken arbitrarily. The effect of key flow parameters like Prandtl number $(\mathrm{Pr})$ non-dimensional temperature profile is depicted through Fig. 2.

It is observed that the fluid temperature decreases for rise in values of Prandtl number. Due to increasing values of Prandtl number, the thermal diffusivity decreases as such the thickness of the thermal boundary layer diminishes. The temperature of fluid particles is thus seen in decreasing trend. 


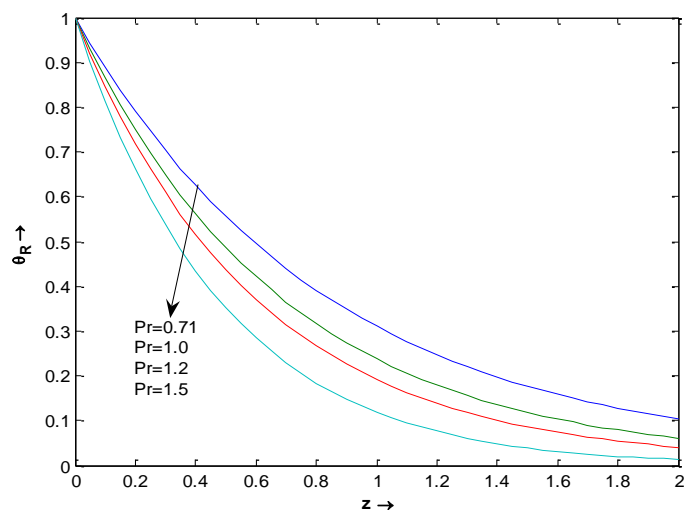

Fig. 2: Graph of temperature $\left(\theta_{R}\right)$ versus normal distance (z) for fixed values of $Q_{s}=0.5, K_{l}=0.5, M=0.8$, $m_{0}=0.2, n=0.5, \delta=0.5, G r=1.5, G m=0.5, \quad \xi=0.5$, $\varepsilon=0.001, \alpha=0.7857, E c=0.02, \omega t=1.57143, u_{p}=0.5$

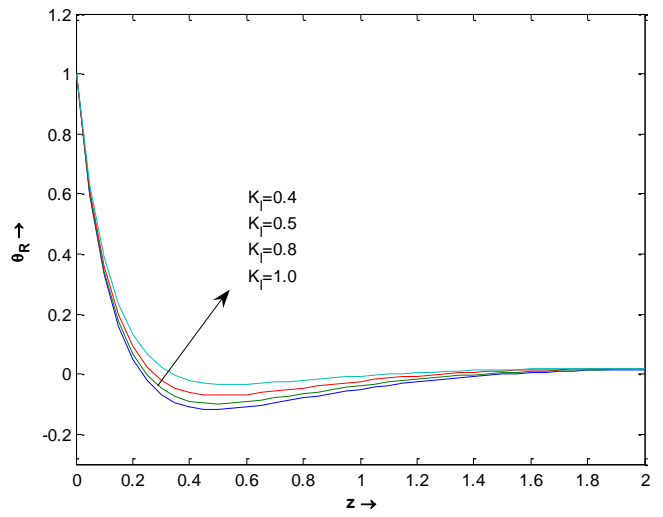

Fig. 3: Graph of temperature $\left(\theta_{R}\right)$ versus normal distance (z) for fixed values of $Q_{s}=0.5$, $M=0.6, \quad m_{0}=0.1, \quad n=0.1, \quad \delta=0.2, \quad G r=3.0$, $G m=3.0, \xi=0.6, \varepsilon=0.001, \alpha=0.7857, E c=0.02$, $\omega t=1.57143, u_{p}=0.5$

The effects of physical parameters such as chemical reaction parameter on the temperature profile $\left(\theta_{R}, z\right)$ are discussed through Fig. 3. The fluid temperature is seen to increase with rise in values of chemical reaction parameter $\left(K_{l}\right)$. The increase in chemical reaction parameter $\left(K_{l}\right)$ causes an increase magnitude of skin friction thereby it helps in rising the amplitude of temperature wave oscillation within the thermal boundary layer. As a consequence, the temperature of fluid particles is observed in an increasing trend. It is observed from the Figs. 23 that fluid temperature obeys the boundary conditions as stated in (2.22-2.23).

The implication of pertinent physical parameters like Schmidt number and chemical reaction parameter on the non-dimensional concentration profile $\left(\phi_{R}, z\right)$ are demonstrated through Figs. 4 and 5 respectively.

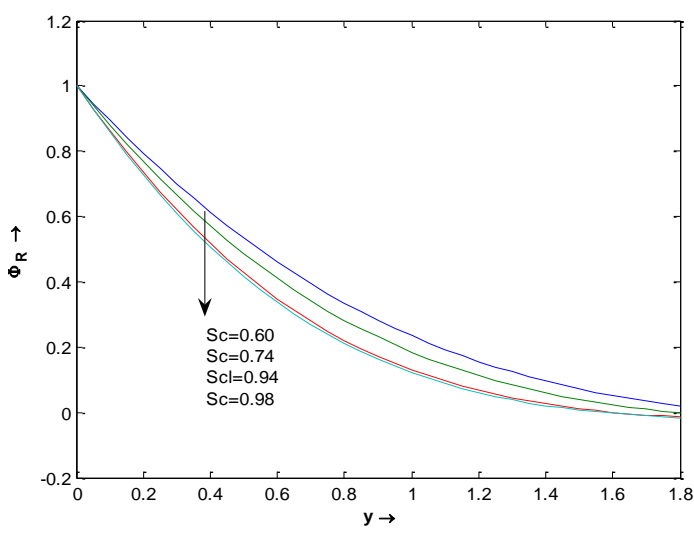

Fig. 4: Graph of concentration $\left(\Phi_{R}\right)$ versus normal distance (z) for fixed values of $Q_{s}=0.3$, $K_{l}=0.5, \quad M=0.8, \quad n=0.4, \quad m_{0}=0.2, \quad \delta=0.3$, $G r=3.5, \quad G m=3.0, \quad K=0.6, \mathrm{~s}=0.2, \quad \varepsilon=0.001$, $\alpha=0.7857, \omega t=1.57143, S r=0.6, u_{P}=0.5$

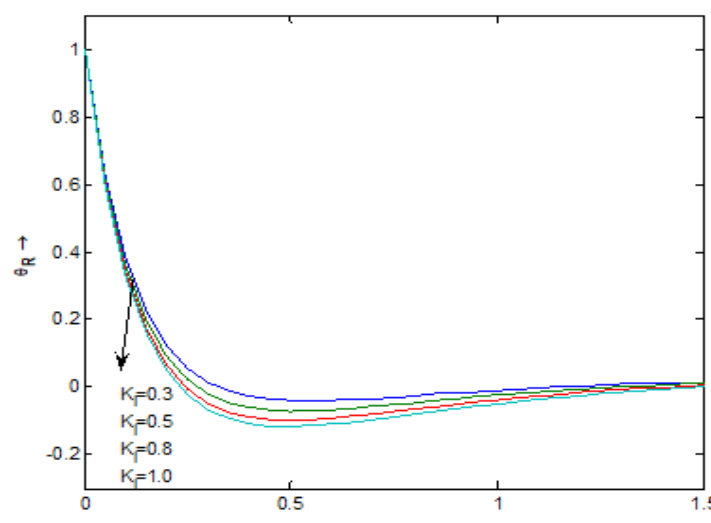

Fig. 5: Graph of concentration $\left(\Phi_{R}\right)$ versus normal distance (z) for fixed values of $Q_{s}=0.3, K_{l}=0.5$, $M=0.8, \quad n=0.4, \quad m_{0}=0.2, \quad \delta=0.3, \quad G r=3.5$, $G m=3.0, \quad K=0.6, \mathrm{~s}=0.2, \quad \varepsilon=0.001, \quad \alpha=0.7857$, $\omega t=1.57143, S r=0.6, u_{P}=0.5$

The concentration of fluid particles is found to decrease for increase in values of Schmidt number as well as chemical reaction parameter. In case of increasing values of Schmidt number, the fluid particles possess low diffusivity. The increasing values of Schmidt number thus helps in decreasing the Brownian mass diffusion effect. This in turn minimizes the fluid concentration within the concentration boundary layer. Due to rise in values of chemical reaction parameter, the constituents from higher concentration region i.e. plate zone diffuse to free stream region. As such, the solutal buoyancy effect reduces near plate zone. From the Figs. 4 and 5, the fluid concentration is thus followed by the boundary conditions as stated in (2.22-2.23) for the fluid concentration. 
Fig. 6 demonstrates the parametric influence of Eckert number on the linear velocity profile $\left(U_{R}, z\right)$. Due to upsurge in values of Eckert number, the linear velocity is seen in an increasing trend. An increase in Eckert number signifies that the kinetic energy is higher than the enthalpy energy. Thus the increasing values of Eckert number causes in increasing the thermal buoyancy force within the thermal boundary layer and thereby accelerates the linear flow rate.

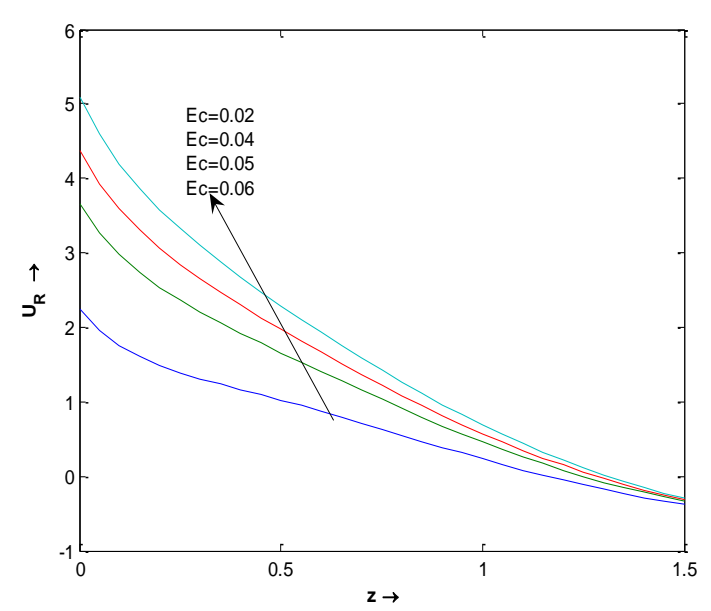

Fig. 6: Graph of linear velocity $\left(u_{R}\right)$ versus normal distance (z) for fixed values of $Q_{s}=0.3$, $K_{l}=0.5, \quad M=0.8, \quad n=0.4, \quad m_{0}=0.2, \quad \delta=0.3$, $G r=1.5, \quad G m=0.8, \quad K=0.6, \xi=0.8, \quad \varepsilon=0.001$, $\alpha=0.7857, \omega t=1.57143, S r=0.6, u_{P}=0.5$

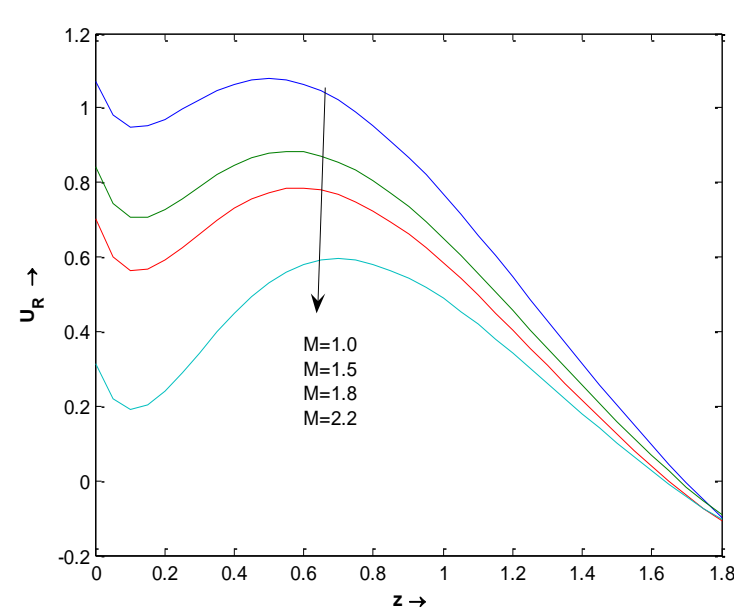

Fig. 7: Graph of linear velocity $\left(u_{R}\right)$ versus normal distance (z) for fixed values of $Q_{s}=0.3, K_{l}=0.5$, $S_{c}=0.60, \quad n=0.4, \quad m_{0}=0.2, \quad \delta=0.3, \quad G r=1.5$, $G m=0.8, \quad K=0.6, \mathrm{~s}=0.2, \quad \varepsilon=0.001, \quad \alpha=0.7857$, $\omega t=1.57143, S r=0.6, u_{P}=0.5, E c=0.01$

The influence of key parameter like magnetic field parameter $(M)$ on linear velocity profile $\left(U_{R}, z\right)$ and angular velocity profile $\left(N_{R}, z\right)$ are demonstrated graphically by Figs. 7 and 8 respectively. It is observed that the linear velocity $\left(U_{R}\right)$ decreases while the angular velocity $\left(N_{R}\right)$ increases with rise in values of magnetic field parameter $(M)$. As the Lorentz force generates within boundary layer flow due to the presence of magnetic field which works in the reverse direction of flow. This retards the linear velocity $\left(N_{R}\right)$ and accelerates the rotational velocity $\left(N_{R}\right)$ of fluid particles. . Furthermore, increasing values of magnetic field parameter increases the resistive forces which accelerate the linear flow rate and decelerate the rotational flow rate.

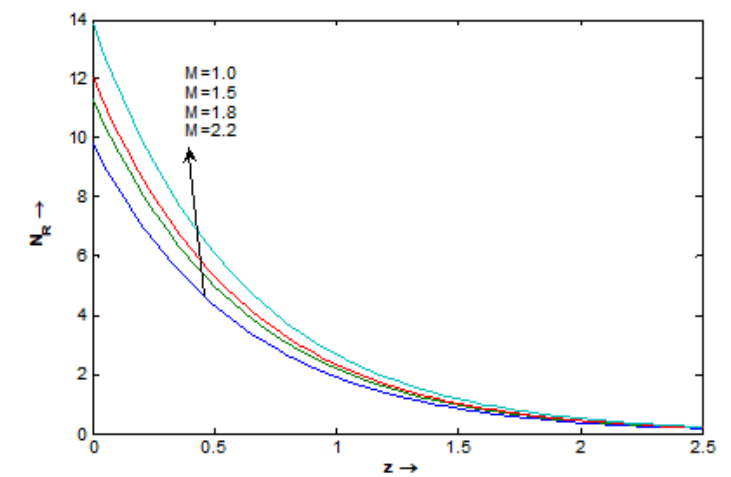

Fig. 8: Graph of angular velocity $\left(N_{R}\right)$ versus normal distance $(\mathrm{z})$ for fixed values of $\xi=0.5, \quad m=0.3, \quad \delta=0.2, \quad n=0.5, \quad G r=0.5$, $G m=1.0, \quad u_{P}=0.5, \quad \varepsilon=0.001, \quad \alpha=0.7857$, $\omega t=1.57143$

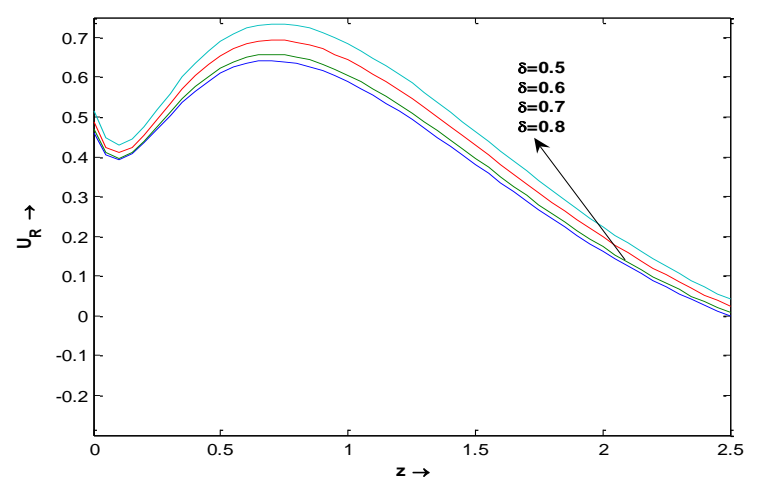

Fig. 9: Graph of linear velocity $\left(u_{R}\right)$ versus normal distance $(z)$ for fixed values of $Q_{s}=0.4, K_{l}=0.4$, $S c=0.60, \quad M=0.6, \quad m_{0}=0.3, \quad G r=0.5, \quad G m=1.8$, $K=0.6, \quad u_{P}=0.5, \quad s=0.2, \quad \varepsilon=0.001, \quad \alpha=0.7857$, $E c=0.02, \omega t=1.57143, S r=0.5$

The effect of gravity modulation parameter $(\delta)$ on linear velocity profile $\left(U_{R}, z\right)$ and the angular velocity profile $\left(N_{R}, z\right)$ are expressed through Figs. 9 and 10 respectively. It is observed that the increasing values of gravity modulation parameter increase the linear velocity whereas decrease the rotational velocity. The increasing values 
of gravitational modulation parameter help in accelerating the linear velocity diffusion rate and decelerating the angular velocity diffusion rate.

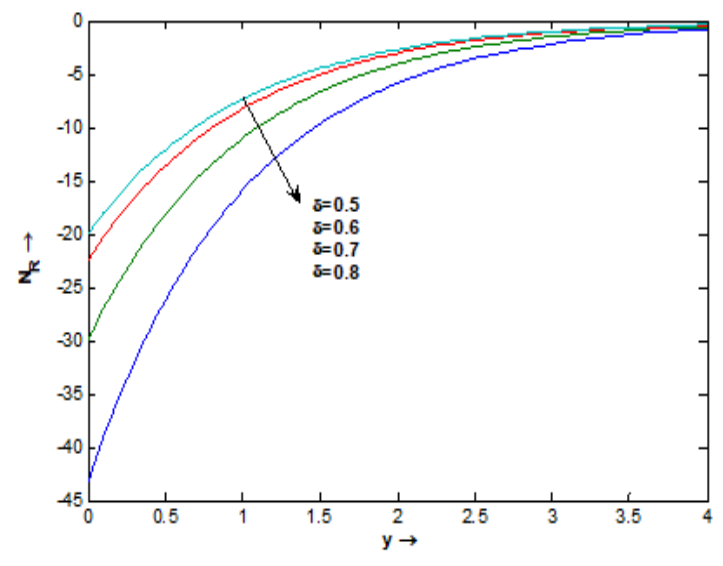

Fig. 10: Graph of angular velocity $\left(N_{R}\right)$ versus normal distance (z) for fixed values of $M=0.8$, $m_{0}=0.2, \quad n=0.3, G r=0.5, \varepsilon=0.001, \alpha=0.7857$, $u_{P}=0.5, \omega t=1.57143, \varepsilon=0.001$

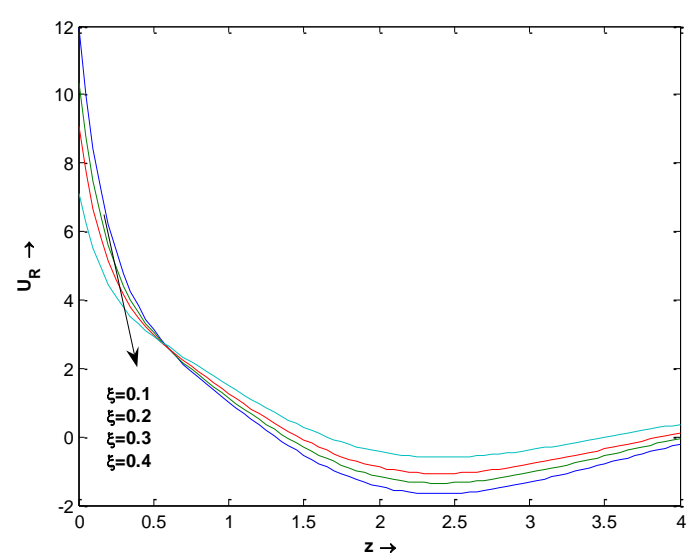

Fig. 11: Graph of linear velocity $\left(u_{R}\right)$ versus normal distance $(z)$ for fixed values of $Q_{s}=0.4$, $K_{l}=0.4, S c=0.60, M=0.6, m_{0}=0.3, \quad G r=0.5$, $G m=1.8, \quad K=0.6, \quad u_{P}=0.5, \quad s=0.2, \quad \varepsilon=0.001$, $\alpha=0.7857, E c=0.02, \omega t=1.57143, S r=0.5$

The physical impact of vortex viscosity parameter $(\xi)$ on the linear velocity profile $\left(U_{R}, z\right)$ and the angular velocity profile $\left(N_{R}, z\right)$ are presented graphically through Figs. 11 and 12. Due to increase in values of vortex viscosity parameter, the angular velocity is found in decreasing trend while a fluctuating trend is viewed for the linear velocity. The increasing values of vortex viscosity parameter decrease the microrotation diffusivity. This increases the linear momentum diffusivity rate and as such the rotational flow rate decelerates and is thus decrease the rotational fluid velocity. But as the linear diffusivity effect near to the plate is less, linear momentum velocity thus takes its minimum value there in and as normal distance increases beyond 0.5 , it shows surprisingly an increasing trend for increasing values of vortex viscosity parameter. It is evident from Figs. 6-12 that both the linear velocity profile $\left(U_{R}, z\right)$ and the angular velocity profile $\left(N_{R}, z\right)$ works in accordance with the boundary conditions as stated in (2.22-2.23).

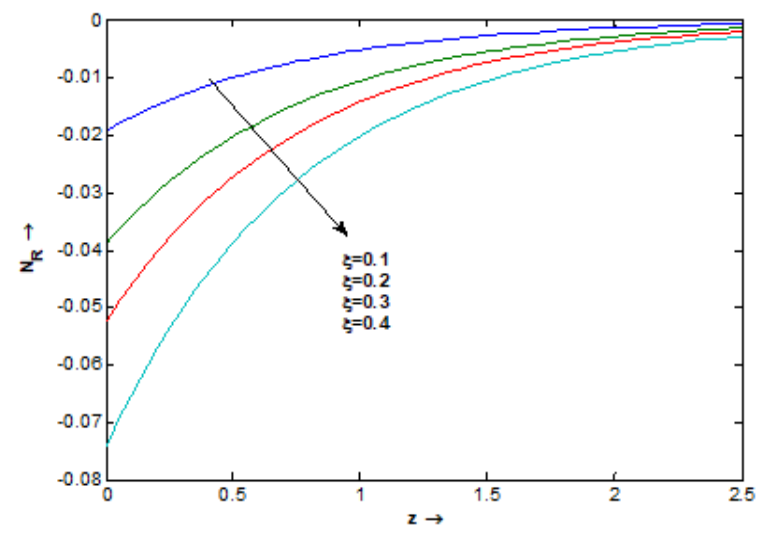

Fig. 12: Graph of angular velocity $\left(N_{R}\right)$ versus normal distance $(z)$ for fixed values of $M=0.8, m_{0}=0.02, \delta=0.3$, $n=0.4, G r=0.5, G m=0.8, \varepsilon=0.001, \alpha=0.7857, u_{P}=0.5, \omega t=1.57143$

In Table 1, a comparative analysis between the Newtonian fluid theory and the micropolar fluid theory is made by taking the parametric variations of heat sink, gravitational modulation parameter and chemical reaction parameter on the skin friction coefficient. It is interesting to observe that the skin friction in micropolar fluids is less than its countering Newtonian fluids. Thus, the micropolar fluid is found suitable to apply in regulating the skin friction effect. 
Table 1: Comparison between micro polar fluid and Newtonian fluid theory for skin friction coefficient $\left(C f_{R}\right)$ with different parameters $t=0.2, \omega=7.857143, \varepsilon=0.01, n=0.5, K_{l}=0.5, \operatorname{Pr}=7.0, S c=0.60, G r=6.0$, $G m=4.0, \psi=0.5236, u_{p}=0.5, \xi=0.5, M=0.5, m_{0}=0.2$.

\begin{tabular}{|l|l|l|l|}
\hline \multicolumn{2}{|l|}{$C f_{R} \rightarrow$} & Newtonian fluid & Micropolar fluid \\
\hline \multirow{3}{*}{$Q_{s}$} & 0.1 & $-3.3949 \times 10^{2}$ & $-9.8048 \times 10^{2}$ \\
\cline { 2 - 4 } & 0.2 & $-3.0033 \times 10^{2}$ & $-9.1279 \times 10^{2}$ \\
\cline { 2 - 4 } & 0.3 & $-2.7196 \times 10^{2}$ & $-8.5805 \times 10^{2}$ \\
\hline \multirow{3}{*}{$K_{l}$} & 0.3 & $-1.4189 \times 10^{3}$ & $-2.3055 \times 10^{3}$ \\
\cline { 2 - 4 } & 0.4 & $-3.5491 \times 10^{3}$ & $-5.2372 \times 10^{3}$ \\
\cline { 2 - 4 } & 0.5 & $-3.7512 \times 10^{3}$ & $-5.0694 \times 10^{3}$ \\
\hline \multirow{4}{*}{$m_{0}$} & 0.3 & $-4.0252 \times 10^{2}$ & $-7.1829 \times 10^{2}$ \\
\cline { 2 - 4 }$\delta$ & 0.4 & $-6.3125 \times 10^{2}$ & $-7.3394 \times 10^{2}$ \\
\cline { 2 - 4 }$\delta$ & 0.6 & $-7.0850 \times 10^{2}$ & $-7.7608 \times 10^{2}$ \\
\hline \multirow{5}{*}{$\delta$} & 1.0 & $-1.4189 \times 10^{3}$ & $-2.3055 \times 10^{3}$ \\
\cline { 2 - 4 } & 1.5 & $-3.5941 \times 10^{3}$ & $-5.2372 \times 10^{3}$ \\
\cline { 2 - 4 } & 3.0 & $-3.7512 \times 10^{3}$ & $-5.0694 \times 10^{3}$ \\
\hline
\end{tabular}

Table 2: Numerical values of skin friction coefficient for fixed values of $\operatorname{Pr}=7.0, S c=0.60, u_{p}=0.5, \omega=7.857143$, $t=0.2, G r=6.0, G m=4.0, n=0.1, \xi=0.5, E c=0.01$

\begin{tabular}{|c|c|c|c|c|c|c|l|}
\hline$\delta$ & $S r$ & $K l$ & $\alpha$ & $\mathrm{Q}_{\mathrm{s}}$ & $M$ & $m_{0}$ & \multicolumn{1}{c|}{$C f_{R}$} \\
\hline 0.3 & 0.1 & 0.3 & 0.5236 & 0.5 & 0.5 & 0.1 & $-3.7772 \times 10^{2}$ \\
\hline 0.3 & 0.1 & 0.5 & 0.5236 & 0.5 & 0.5 & 0.1 & $-2.6469 \times 10^{2}$ \\
\hline 0.4 & 0.1 & 0.2 & 0.5236 & 0.5 & 0.5 & 0.1 & $-5.4020 \times 10^{2}$ \\
\hline 0.5 & 0.1 & 0.2 & 0.5236 & 0.5 & 0.5 & 0.1 & $-6.3018 \times 10^{2}$ \\
\hline 0.3 & 0.2 & 0.2 & 0.5236 & 0.5 & 0.5 & 0.1 & $-4.5432 \times 10^{2}$ \\
\hline 0.3 & 0.5 & 0.2 & 0.5236 & 0.5 & 0.5 & 0.1 & $-4.1895 \times 10^{2}$ \\
\hline 0.3 & 0.1 & 0.2 & 0.5236 & 0.5 & 0.5 & 0.1 & $-4.6611 \times 10^{2}$ \\
\hline 0.3 & 0.1 & 0.2 & 0.7857 & 0.5 & 0.5 & 0.1 & $-4.2230 \times 10^{2}$ \\
\hline 0.3 & 0.1 & 0.2 & 1.0472 & 0.5 & 0.5 & 0.1 & $-3.6827 \times 10^{2}$ \\
\hline 0.3 & 0.1 & 0.2 & 0.5236 & 0.3 & 0.5 & 0.1 & $-4.5634 \times 10^{2}$ \\
\hline 0.3 & 0.1 & 0.2 & 0.5236 & 0.4 & 0.5 & 0.1 & $-4.6071 \times 10^{2}$ \\
\hline 0.3 & 0.1 & 0.2 & 0.5236 & 0.5 & 0.6 & 0.1 & $-4.7792 \times 10^{2}$ \\
\hline 0.3 & 0.1 & 0.2 & 0.5236 & 0.5 & 0.7 & 0.1 & $-4.7524 \times 10^{2}$ \\
\hline 0.3 & 0.1 & 0.2 & 0.5236 & 0.5 & 0.5 & 0.3 & $-4.6665 \times 10^{2}$ \\
\hline 0.3 & 0.1 & 0.2 & 0.5236 & 0.5 & 0.5 & 0.4 & $-4.6755 \times 10^{2}$ \\
\hline
\end{tabular}

The numerical data relating to the varied values of co-efficient of skin-friction $\left(C f_{R}\right)$ and couple stress coefficient $\left(\mathrm{Cm}_{R}\right)$ for arbitrary change in values of various parameters like $\delta, S r, K_{l}, \alpha, Q_{s}, M, m$ (on $\left.C f_{R}\right)$ and $\delta, \xi, N, M, \alpha, G r, G m$ (on $C m_{R}$ ) are presented through Tables 2 and 3 respectively. It is worth full in perceiving that the skin friction coefficient and couple stress coefficient are being influenced by the increasing values of varied key parameters. It is observed that, $C f_{R}$ increases only due to increase in values of $\delta, Q_{s}$ while $C m_{R}$ increases in presence of $\delta, N, \alpha, G r$ and $G m$. A fluctuating effect on $C f_{R}$ and $C m_{R}$ is noted in presence of $M$. 
Table 3: Numerical values of couple stress coefficient for fixed values of $\operatorname{Pr}=7.0, S c=0.60, u_{p}=0.5, \omega=7.857143$, $t=0.2, n=0.1, m_{0}=0.2, \varepsilon=0.01$

\begin{tabular}{|l|l|l|l|l|l|l|l|}
\hline$\delta$ & $\xi$ & $N$ & $M$ & $\alpha$ & \multicolumn{1}{|c|}{$G r$} & \multicolumn{1}{|c|}{$\mathrm{Gm}$} & \multicolumn{1}{c|}{$\mathrm{Cm}_{\mathcal{R}}$} \\
\hline 0.3 & 0.2 & 0.2 & 0.5 & 0.5236 & 6.0 & 5.0 & 7.4592 \\
\hline 0.3 & 0.3 & 0.2 & 0.5 & 0.5236 & 6.0 & 5.0 & 5.1062 \\
\hline 0.2 & 0.3 & 0.2 & 0.5 & 0.5236 & 6.0 & 5.0 & 5.1060 \\
\hline 0.4 & 0.3 & 0.2 & 0.5 & 0.5236 & 6.0 & 5.0 & 5.1066 \\
\hline 0.3 & 0.3 & 0.3 & 0.5 & 0.5236 & 6.0 & 5.0 & 6.9266 \\
\hline 0.3 & 0.3 & 0.4 & 0.5 & 0.5236 & 6.0 & 5.0 & 8.1445 \\
\hline 0.3 & 0.3 & 0.2 & 0.2 & 0.5236 & 6.0 & 5.0 & 5.0953 \\
\hline 0.3 & 0.3 & 0.2 & 0.4 & 0.5236 & 6.0 & 5.0 & 5.1015 \\
\hline 0.3 & 0.3 & 0.2 & 0.5 & 0.0000 & 6.0 & 5.0 & 5.0932 \\
\hline 0.3 & 0.3 & 0.2 & 0.5 & 0.5236 & 6.0 & 5.0 & 5.1062 \\
\hline 0.3 & 0.3 & 0.2 & 0.5 & 0.7857 & 6.0 & 5.0 & 5.1193 \\
\hline 0.3 & 0.3 & 0.2 & 0.5 & 1.0472 & 6.0 & 5.0 & 5.1324 \\
\hline 0.3 & 0.3 & 0.2 & 0.5 & 0.5236 & 4.0 & 5.0 & 4.1802 \\
\hline 0.3 & 0.3 & 0.2 & 0.5 & 0.5236 & 5.0 & 5.0 & 4.6432 \\
\hline 0.3 & 0.3 & 0.2 & 0.5 & 0.5236 & 6.0 & 6.0 & 5.5693 \\
\hline 0.3 & 0.3 & 0.2 & 0.5 & 0.5236 & 6.0 & 4.0 & 4.6432 \\
\hline
\end{tabular}

Table 4: Numerical values of Nusselt number for fixed values of $\operatorname{Pr}=7.0, S c=0.60, u_{p}=0.5, \omega=7.857143, t=0.2$, $n=0.1, \xi=0.5, E c=0.01, \varepsilon=0.01$.

\begin{tabular}{|l|l|l|l|l|l|l|l|l|l|}
\hline$K_{l}$ & $Q_{s}$ & $\alpha$ & $E c$ & $\delta$ & $M$ & $m_{0}$ & $G r$ & $G m$ & $N u_{R}$ \\
\hline 0.3 & 0.5 & 0.5236 & 0.01 & 0.2 & 0.5 & 0.1 & 6.0 & 4.0 & 2.3496 \\
\hline 0.5 & 0.5 & 0.5236 & 0.01 & 0.2 & 0.5 & 0.1 & 6.0 & 4.0 & 2.1508 \\
\hline 0.3 & 0.5 & 0.5236 & 0.01 & 0.2 & 0.3 & 0.1 & 6.0 & 4.0 & 2.2676 \\
\hline 0.3 & 0.5 & 0.5236 & 0.01 & 0.2 & 0.4 & 0.1 & 6.0 & 4.0 & 2.3126 \\
\hline 0.3 & 0.3 & 0.5236 & 0.01 & 0.2 & 0.5 & 0.1 & 6.0 & 4.0 & 1.7267 \\
\hline 0.3 & 0.4 & 0.5236 & 0.01 & 0.2 & 0.5 & 0.1 & 6.0 & 4.0 & 2.0325 \\
\hline 0.3 & 0.5 & 0.5236 & 0.01 & 0.2 & 0.5 & 0.1 & 5.0 & 4.0 & 1.9215 \\
\hline 0.3 & 0.5 & 0.5236 & 0.01 & 0.2 & 0.5 & 0.1 & 4.0 & 4.0 & 2.1267 \\
\hline 0.3 & 0.5 & 0.5236 & 0.01 & 0.2 & 0.5 & 0.1 & 6.0 & 5.0 & 3.6701 \\
\hline 0.3 & 0.5 & 0.5236 & 0.01 & 0.2 & 0.5 & 0.1 & 6.0 & 6.0 & 5.7318 \\
\hline 0.3 & 0.5 & 0.5236 & 0.01 & 0.1 & 0.5 & 0.1 & 6.0 & 4.0 & 1.5029 \\
\hline 0.3 & 0.5 & 0.5236 & 0.01 & 0.3 & 0.5 & 0.1 & 6.0 & 4.0 & 4.7200 \\
\hline 0.3 & 0.5 & 0.5236 & 0.01 & 0.2 & 0.5 & 0.2 & 6.0 & 4.0 & 2.4200 \\
\hline 0.3 & 0.5 & 0.5236 & 0.01 & 0.2 & 0.5 & 0.3 & 6.0 & 4.0 & 2.4795 \\
\hline 0.3 & 0.5 & 0.0000 & 0.01 & 0.2 & 0.5 & 0.1 & 6.0 & 4.0 & 2.1761 \\
\hline 0.3 & 0.5 & 0.7857 & 0.01 & 0.2 & 0.5 & 0.1 & 6.0 & 4.0 & 2.4873 \\
\hline 0.3 & 0.5 & 1.0472 & 0.01 & 0.2 & 0.5 & 0.1 & 6.0 & 4.0 & 2.5827 \\
\hline 0.3 & 0.5 & 0.5236 & 0.02 & 0.2 & 0.5 & 0.1 & 6.0 & 4.0 & 3.8528 \\
\hline 0.5 & 0.5 & 0.5236 & 0.03 & 0.2 & 0.5 & 0.1 & 6.0 & 4.0 & 5.3560 \\
\hline
\end{tabular}


In Tables 4 and 5 , the numerical values of Nusselt number $\left(N u_{R}\right)$ as well as Sherwood number $\left(S h_{R}\right)$ are computed for various values of $\delta, \alpha, Q_{s}, M, m, G r, G m \quad\left(\right.$ on $\left.N u_{R}\right)$. and $m, n, \delta, \quad S r, \quad K_{l}$, $u_{p}, Q_{s}, M\left(\right.$ on $\left.S h_{R}\right)$. The values of $N u_{R}$ is found to increase due to positive increment in values of $\delta, \alpha, G r, G m$ whereas $S h_{R}$ increases due to presence of $n, \delta, \alpha, M$. The computed values of Nusselt number as well as Sherwood number is thus found to be regulated by the governed flow parameters.

Table 5: Numerical values of Sherwood number for fixed values of $\operatorname{Pr}=7.0, S c=0.60, u_{p}=0.5, \omega=7.857143, t=0.2$, $G r=6.0, G m=4.0, n=0.1, \xi=0.5, E c=0.001, \varepsilon=0.001$.

\begin{tabular}{|l|l|l|l|l|l|l|l|l|l|}
\hline$m_{0}$ & $n$ & $M$ & $K_{l}$ & $Q_{s}$ & $u_{p}$ & $S r$ & $\delta$ & $\alpha$ & $S h_{R}$ \\
\hline 0.1 & 0.1 & 0.5 & 0.3 & 1.1 & 0.5 & 0.3 & 0.1 & 0.5236 & $4.2154 \times 10^{4}$ \\
\hline 0.1 & 0.1 & 0.5 & 0.3 & 1.1 & 0.5 & 0.3 & 0.3 & 0.5236 & $6.0749 \times 10^{4}$ \\
\hline 0.2 & 0.1 & 0.5 & 0.3 & 1.1 & 0.5 & 0.3 & 0.2 & 0.5236 & $5.0631 \times 10^{4}$ \\
\hline 0.3 & 0.1 & 0.5 & 0.3 & 1.1 & 0.5 & 0.3 & 0.2 & 0.5236 & $5.0630 \times 10^{4}$ \\
\hline 0.1 & 0.1 & 0.7 & 0.3 & 1.1 & 0.5 & 0.3 & 0.2 & 0.5236 & $5.0633 \times 10^{4}$ \\
\hline 0.1 & 0.1 & 0.9 & 0.3 & 1.1 & 0.5 & 0.3 & 0.2 & 0.5236 & $5.0644 \times 10^{4}$ \\
\hline 0.1 & 0.3 & 0.5 & 0.3 & 1.1 & 0.5 & 0.3 & 0.2 & 0.5236 & $5.0665 \times 10^{4}$ \\
\hline 0.1 & 0.4 & 0.5 & 0.3 & 1.1 & 0.5 & 0.3 & 0.2 & 0.5236 & $5.0699 \times 10^{4}$ \\
\hline 0.1 & 0.1 & 0.5 & 0.4 & 1.1 & 0.5 & 0.3 & 0.2 & 0.5236 & $5.0602 \times 10^{4}$ \\
\hline 0.1 & 0.1 & 0.5 & 0.6 & 1.1 & 0.5 & 0.3 & 0.2 & 0.5236 & $5.0547 \times 10^{4}$ \\
\hline 0.1 & 0.1 & 0.5 & 0.3 & 0.8 & 0.5 & 0.3 & 0.2 & 0.5236 & $4.9813 \times 10^{4}$ \\
\hline 0.1 & 0.1 & 0.5 & 0.3 & 1.3 & 0.5 & 0.3 & 0.2 & 0.5236 & $5.1272 \times 10^{4}$ \\
\hline 0.1 & 0.1 & 0.5 & 0.3 & 1.1 & 0.5 & 0.1 & 0.2 & 0.5236 & $5.0632 \times 10^{4}$ \\
\hline 0.1 & 0.1 & 0.5 & 0.3 & 1.1 & 0.5 & 0.4 & 0.2 & 0.5236 & $5.0630 \times 10^{4}$ \\
\hline 0.1 & 0.1 & 0.5 & 0.3 & 1.1 & 0.5 & 0.3 & 0.2 & 0.7857 & $5.0634 \times 10^{4}$ \\
\hline 0.1 & 0.1 & 0.5 & 0.3 & 1.1 & 0.5 & 0.3 & 0.2 & 1.0472 & $5.0639 \times 10^{4}$ \\
\hline
\end{tabular}

In Table 6, a comparative study of the present model with the work of Deka and Soundalgekar(2006) has been made to compare the numerical values of skin friction for both the case of Prandtl number as 0.71 and 7.0 . From the table, it is found that the skin friction effects in both the works are found to be decreasing due to increasing values of $\omega t$ for fixed values of certain physical parameters $G r=1.0, \delta=1.0, t=2.0, \varepsilon=0.2$. The range of values obtained are found to be close to one another in the respective papers thus justifying a good agreement of the present work to that work of Deka and Soundalgekar.

Table 6: Numerical values of skin friction for various values of physical parameters when $t=2.0, G r=1.0, \varepsilon=0.2$, $\delta=1.0$.

\begin{tabular}{|l|l|l|l|l|l|}
\hline$\omega$ & $\omega t$ & $\begin{array}{l}\text { Pr =0.71 (Present } \\
\text { paper) }\end{array}$ & $\begin{array}{l}\text { Pr =7.0 } \\
\text { Present paper) }\end{array}$ & $\begin{array}{l}\text { Pr =0.71 Deka and } \\
\text { Sondalgekar } \\
(2006) \text { paper }\end{array}$ & $\begin{array}{l}\text { Pr =7.0 Deka and } \\
\text { Sondalgekar } \\
\text { (2006)paper }\end{array}$ \\
\hline 5 & 0 & 1.4755 & 0.1667 & 0.5605 & 0.4211 \\
\hline 5 & $\pi / 6$ & 1.4934 & 0.1659 & 1.9936 & 1.8359 \\
\hline 5 & $\pi / 4$ & 1.4885 & 0.1631 & 1.4204 & 1.2692 \\
\hline 5 & $\pi / 3$ & 1.4621 & 0.1590 & 1.1337 & 0.9865 \\
\hline 5 & $\pi / 2$ & 1.4621 & 0.1477 & 0.8471 & 0.7038 \\
\hline 10 & $\pi / 4$ & 1.4694 & 0.1621 & 0.8539 & 0.7027 \\
\hline 15 & $\pi / 4$ & 1.4585 & 0.1612 & 0.6492 & 0.4979 \\
\hline
\end{tabular}




\section{Conclusion}

A theoretical investigation on free convection flow of unsteady MHD micropolar fluid past an inclined plate with the effects of gravitational modulation, Hall effect, Joule heating and heat sink. The investigation reveals that the fluid temperature decreases due to increase in values of Prandtl number. The concentration of fluid particles decreases with rise in values of flow parameters like Schmidt number and chemical reaction. The linear fluid velocity is found to increases due to increasing values of gravitational modulation parameter, Eckert number, but decreases for increasing values of magnetic field parameter. The rotational velocity of fluid particles is found increasing for rise in values of magnetic field parameter while it decreases for increasing values of gravitational modulation parameter. The skin friction effect is possible to regulate by using the micropolar fluid theory.

\section{References}

Alam, M. S. and Hossain, S. M.(2013): A new similarity approach for an unsteady two-dimensional forced convective flow of a micropolar fluid along a wedge, International Journal of Applied Mathematics and Mechanics, Vol. 9, No.14, pp. 75 - 89.

Alam, M. S. and Rahman, M. M. (2005): Dufour and Soret effects on MHD free convective heat and mass transfer flow past a vertical porous flat plate embedded in a porous medium, Journal of Naval Architecture and Marine Engineering, Vol. 2, No.1, pp. 55-65. https://doi.org/10.3329/jname.v2i1.2030

Alam, M. S., Sattar, M. A., Rahman, M. M.C. and Postelnicu, A.(2010): Local similarity solution of an unsteady two-dimensional MHD convective flow of a micropolar fluid past a continuously moving porous plate under the influence of magnetic field, International Journal Heat and Technology, Vol. 28, No.2, pp. 95-105.

Ayano, M. S. and Demeke, N.T. (2015): Hall effect and temperature distribution on unsteady micro polar fluid flow in a moving walls. International Journal of Sciences: Basic and Applied Research , Vol.24, No.7, pp.60-75. http://gssrr.org/index.php?journal=JournalOfBasicAndApplied

Chamkha, A. J. (1997): MHD-free convection from a vertical plate embedded in a thermally stratified porous medium with Hall effects, Applied Mathematical Modelling, Vol. 21, No.10, pp.603-609.

https://doi.org/10.1016/S0307-904X(97)00084-X

Chamkha, A. J., Mohamed, R. A. \& Ahmed, S. E. (2011): Unsteady MHD natural convection from a heated vertical porous plate in a micropolar fluid with Joule heating, chemical reaction and radiation effects, Meccanica, Vol.46, No.2, pp. 399-411. https://doi.org/10.1007/s11012-010-9321-0

Chamkha, A. J., Rashad, A. M., Mansour, M. A., Armaghani, T. \& Ghalambaz, M. (2017): Effects of heat sink and source and entropy generation on MHD mixed convection of a $\mathrm{Cu}$-water nanofluid in a lid-driven square porous enclosure with partial slip, Physics of Fluids, Vol. 29, No.5, pp.052001. https://doi.org/10.1063/1.4981911

Eldabe, N. T. and Ouaf, M. E. (2006): Chebyshev finite difference method for heat and mass transfer in a hydromagnetic flow of a micropolar fluid past a stretching surface with Ohmic heating and viscous dissipation, Applied Mathematics and Computation, Vol. 177, No.2, pp.561-571. https://doi.org/10.1016/j.amc.2005.07.071

Eringen, A. C. (1966): Theory of micropolar fluids, Journal of Mathematics and Mechanics, Vol. 16, pp.1-18. http://dx.doi.org/10.1512/iumj.1967.16.16001

Gorla, R. S. R., \& Chamkha, A. (2011): Natural convective boundary layer flow over a nonisothermal vertical plate embedded in a porous medium saturated with a nanofluid, Nanoscale and Microscale Thermophysical Engineering, Vol.15 No.2, pp.81-94. https://doi.org/10.1080/15567265.2010.549931

Hayat, T., yasmin, H., Ahmad, B. and Chen, G. Q. (2014): Exact solution for peristaltic transport of a micropolar fluid in a channel with convective boundary conditions and heat source/sink, Zeitschrift für Naturforschung A, Vol. 69, No. 8-9, pp. 425-432. https://doi.org/10.5560/zna.2014-0038

Khedr, M. E. M., Chamkha, A. J. and Bayomi, M. (2009): MHD flow of a micropolar fluid past a stretched permeable surface with heat generation or absorption, Nonlinear Analysis: Modelling and Control, Vol. 14, No.1, pp. 27-40. https://doi.org/10.15388/NA.2009.14.1.14528

Kishan, N. and Amrutha, P.(2010): Effects of viscous dissipation on MHD flow with heat and mass transfer over a stretching surface with heat source, thermal stratification and chemical reaction, Journal of Naval Architecture and Marine Engineering,Vol.7, No.1, pp.11-18. https://doi.org/10.3329/jname.v7i1.3254

Koriko, O. K., Omowaye, A. J., Animasaun, I. L. and Bamisaye, M. E. (2017): Melting heat transfer and induced-magnetic field effects on the micropolar fluid flow towards stagnation point: Boundary layer analysis, International Journal of Engineering Research in Africa, Vol. 29, pp.10-20.

https://doi.org/10.4028/www.scientific.net/JERA.29.10

Kumar, A. \& Pranesh, S. (2018): Study of heat transfer in a time dependent vertically oscillating micropolar liquid, Recent Trends in Fluid Mechanics, Vol. 5 No.1, pp. 63-74. https://doi.org/10.37591/rtfm.v5i1.908 
Narayana, P. S., Venkateswarlu, B. and Venkataramana, S. (2013): Effects of Hall current and radiation absorption on MHD micropolar fluid in a rotating system, Ain Shams Engineering Journal, Vol. 4, No.4, pp.843854. https://doi.org/10.1016/j.asej.2013.02.002

Pattnaik, P, Jena, S., Dei, A. and Sahu, G. (2019). Impact of chemical reaction on micropolar fluid past a stretching sheet, JP Journal of Heat and Mass Transfer. Vol.18. pp.207-223.

http://dx.doi.org/10.17654/HM018010207

Pranesh, S., Tarannum, S. and Joseph, T. V. (2014): Linear and Weakly Non-Linear Analyses of Gravity Modulation and Electric Field on the onset of Rayleigh-Bénard Convection in a Micropolar Fluid, Journal of Advances in Mathematics, Vol. 9, No.3, pp. 2057-2082. https://doi.org/10.24297/jam.v9i3.2426

Rajvanshi, S. C., Saini, B. S. and Bhawn, Jeet (2014): Effect of radiation and gravity modulation on unsteady MHD free convection flow through porous medium in slip-flow regime with entropy, Walailak Journal of Science and Technology (WJST), Vol. 11, No.3, pp.225-242. https://doi.org/10.14456/WJST.2014.41

Rajput, U.S. and Kumar,G.(2019):Effects of radiation and chemical reaction on MHD flow past a vertical plate with variable temperature and mass diffusion, Journal of Naval Architecture and Marine Engineering,Vol.16, No.2, pp.99-108. https://doi.org/10.3329/jname.v16i2.29526

Reddy, K. S. N., Babu, M. S., Varma, S. V. K. and Reddy, N. B. (2014): Hall current and dufour effects on MHD flow of a micropolar fluid past a vertical plate in the presence of radiation absorption and chemical reaction, IOSR Journal of Mathematics, pp.106-121. https://doi.org/10.9790/5728-1041106121

Rout, P. K., Sahoo, S. N., Dash, G. C., \& Mishra, S. R. (2016): Chemical reaction effect on MHD free convection flow in a micropolar fluid, Alexandria Engineering Journal, Vol.55, No.3, pp.2967-2973.

https://doi.org/10.1016/j.aej.2016.04.033

Shamshuddin, M. D., Mishra, S. R., Bég, O. A. and Kadir, A. (2018): Unsteady reactive magnetic radiative micropolar flow, heat and mass transfer from an inclined plate with joule heating, A model for magnetic polymer processing. Proceedings of the Institution of Mechanical Engineers, Journal of Mechanical Engineering Science, https://doi.org/10.1177/0954406218768837

Sharidan, S., Amin, N. and Pop, I. (2005): G-jitter induced free convection near a two-dimensional stagnation point in micropolar fluids, International Journal of Applied Mechanics and Engineering, Vol.10, No.2, pp.311328.

Sengupta, S. and Sen, N. (2013): Free convective heat and mass transfer flow past an oscillating plate with heat generation, thermal radiation and thermo-diffusion effects, J.P. Journal of Heat and Mass Transfer, Vol. 8, No.2, pp.187.

Sengupta, S. and Ahmed, N. (2014): MHD free convective chemically reactive flow of a dissipative fluid with thermal-diffusion, fluctuating wall temperature and concentrations in velocity slip regime, International Journal of Applied Maths and Mechanics Vol.10, No.4, pp.27-54.

Sengupta, S. and Ahmed, N. (2016): MHD free convective mass transfer flow of radiative uniform heat generation (absorption) fluid through a wavy permeable channel in the presence of Soret and Dufour effects, Canadian Journal of Physics, Vol. 95, No.1, pp. 44-58. https://doi.org/10.1139/cjp-2014-0599

Shamshuddin, M. D., Chamkha, A. J., Raju, M. C. and Thumma, T. (2018):Computation of unsteady MHD mixed convective heat and mass transfer in dissipative reactive micropolar flow considering Soret and Dufour effects. Frontiers in Heat and Mass Transfer, Vol.10. https://doi.org/10.5098/hmt.10.15.

Singh, B. (2017): Hall effect on MHD flow of visco-elastic micro-polar fluid layer heated from below saturating a porous medium, International Journal of Engineering, Science and Technology, Vol.9, No.4, pp.48-66. https://doi.org/10.4314/ijest.v9i4.5

Sreenivasulu, P., Poornima, P. and Bhaskar Reddy N.B (2018): Internal heat generation effect on radiation heat transfer MHD dissipating flow of a micropolar fluid with variable wall heat flux, Journal of Naval Architecture and Marine Engineering, Vol.15 No.1, pp.53-64. https://doi.org/10.3329/jname.v15i1.19582

Takhar, H. S., Chamkha, A. J. and Nath, G. (2001): Unsteady three-dimensional MHD-boundary-layer flow due to the impulsive motion of a stretching surface, Acta Mechanica, Vol.146 No.1-2, pp.59-71. http://www.springerlink.com/content/hwh002v742627x...

Umavathi, J. C. and Sultana, J. (2012): Mixed convection flow of a micropolar fluid with concentration in a vertical channel in the presence of heat source or sink, International Journal of Mathematical Archive, Vol. 3, No.10,pp.3556-3569.

Yadav, P. K., Jaiswal, S., Asim, T. and Mishra, R. (2018): Influence of a magnetic field on the flow of a micropolar fluid sandwiched between two Newtonian fluid layers through a porous medium, The European Physical Journal Plus, Vol.133, No.7, pp.247. https://doi.org/10.1140/epip/i2018-12071-5

Yekasi, V., Pranesh, S. and Bathul, S. (2016): Effects of gravity modulation and internal heat generation on the onset of Rayleigh-Bènard convection in a micropolar fluid, Journal of Advances in Mathematics, Vol.12, No.6, pp.6270-6285. https://doi.org/10.24297/jam.v12i6.3829 